\title{
Phosphorylation-dependent routing of RLP44 towards brassinosteroid or phytosulfokine signalling
}

Borja Garnelo Gómez ${ }^{1,2}$, Rosa Lozano-Durán², Sebastian Wolf ${ }^{1 *}$

${ }^{1}$ Centre for Organismal Studies Heidelberg, University of Heidelberg, INF230, 69120 Heidelberg, Germany

${ }^{2}$ Shanghai Center for Plant Stress Biology, CAS Center for Excellence in Molecular Plant Sciences, Chinese Academy of Sciences (CAS), Shanghai 201602 China

*for correspondence: sebastian.wolf@cos.uni-heidelberg.de

\begin{abstract}
Plants rely on a complex network of cell surface receptors to integrate developmental and environmental cues into behaviour adapted to the conditions. The largest group of these receptors, leucine-rich repeat receptor-like kinases, form a complex interaction network that is modulated and extended by receptor-like proteins. This raises the question of how specific outputs can be generated when receptor proteins are engaged in a plethora of promiscuous interactions. RECEPTOR-LIKE PROTEIN 44 acts to promote brassinosteroid and phytosulfokine signalling, which orchestrate a wide variety of cellular responses. However, it is unclear how these activities are coordinated. Here, we show that RLP44 is phosphorylated in its highly conserved $\mathrm{C}$-terminal cytosolic tail and that this post-translational modification governs its subcellular localization. RLP44 variants in which phosphorylation is blocked enter endocytosis prematurely, leading to an almost entirely intracellular localization, whereas mimicking phosphorylation results in preferential RLP44 localization at the plasma membrane. Phosphorylation is crucial for regulating RLP44's interaction with the brassinosteroid receptor BRASSINOSTEROID INSENSITIVE 1, and thus its function in BR signalling activation. In contrast, the interaction of RLP44 with PHYTOSULFOKINE RECEPTOR 1 is not affected by its phospho-status. Analysis of the contribution of individual amino acid modifications suggests that routing of RLP44 to its target receptor complexes is controlled by its phosphorylation pattern, providing a framework to understand how a common component of different receptor complexes can get specifically engaged in a particular signalling pathway.
\end{abstract}




\section{Introduction}

To integrate environmental cues with intrinsic developmental programs, plants depend on the perception of extracellular signals by their expanded family of cell surface receptors. The largest group of these plasma membrane-localized proteins is formed by leucine-rich repeat receptor-like kinases (LRR-RLKs), characterized by the presence of a signal peptide for entry into the secretory pathway, a leucine-rich repeat containing extracellular domain, a single pass transmembrane domain, and an intracellular kinase domain with homology to animal Irak/Pelle proteins (Shiu and Bleecker, 2001). LRR-RLKs form an extensive interaction network reflecting the need to decipher complex information about the environment (SmakowskaLuzan et al., 2018). LRR-RLPs, which resemble LRR-RLKs, but lack a kinase domain, contribute to RLK signalling in a variety of ways (Jamieson et al., 2018), further expanding the complexity of the network. Thus, the multitude of potential interactions and extensive sharing of components between pathways, raise a central question in plant signal transduction: how can distinct signalling responses be achieved? A partial answer is provided by the discovery of membrane sub-compartmentalization that helps to spatially separate potential interaction partners (Jarsch et al., 2014, Wang et al., 2015b, Bucherl et al., 2017, Gronnier et al., 2017, Ott, 2017). Furthermore, selective endocytosis, recycling, and eventually, the degradation of RLKs in the vacuole (Martins et al., 2015, Mbengue et al., 2016, Zhou et al., 2018) are crucial for tuning of signalling. Most, if not all plasma membrane receptors described so far undergo endocytosis in vesicles coated by clathrin, which depend on cytosolic adaptor complexes for cargo selection and transport (Van Damme et al., 2011, Di Rubbo et al., 2013, Gadeyne et al., 2014). In some, but not all, cases ligand binding promotes internalization, possibly to establish a refractory phase after ligand exposure and prevent potentially harmful continuous activation (Robatzek et al., 2006, Nimchuk et al., 2011, Ortiz-Morea et al., 2016). Endocytic trafficking of LRR-RLK proteins is intimately linked to post-translational modification of their cytosolic domains through addition of the small protein ubiquitin to lysine residues (Dubeaux and Vert, 2017). A different kind of post-translational modification, phosphorylation, is at the core of RLK signalling regulation. Binding of extracellular ligands to the ectodomains of several LRR-RLKs has been shown to mediate hetero-dimerization with a shape-complimentary co-receptor of the SOMATIC-EMBRYOGENESIS RECEPTOR KINASE (SERK) family (Couto and Zipfel, 2016, Hohmann et al., 2017, Mithoe and Menke, 2018), juxtaposing their kinase domains in the cytosol. This, in turn, leads to auto and trans-phosphorylation of the kinases, resulting in an activated receptor complex capable of recruiting and phosphorylating downstream signalling components. Recent evidence suggests differential requirements for individual phosphorylation events in SERK kinase domains depending on the interacting RLK (Perraki et al., 2018). Signalling output is further modified by the activity of phosphatases and the phosphorylation-dependent release of inhibitory factors (Park et al., 2008, Jaillais et al., 2011b, Lin et al., 2013, Monaghan et al., 2014, Couto et al., 2016). In summary, post-translational modification through phosphorylation and ubiquitination are key mechanisms to spatially and temporally control LRR-RLK-mediated signalling. One of the best-characterized LRR-RLKs is the receptor for brassinosteroid (BR) phytohormones, BRASSINOSTEROID INSENSITIVE 1 (BRI1) (Li and Chory, 1997). Signalling mediated by BRI1 and its co-receptors, such as SOMATIC-EMBRYOGENESIS RECEPTOR KINASE 1/BRI1-ASSOCIATED KINASE1 (SERK3/BAK1) (Li et al., 2002, Nam and Li, 2002), plays a crucial role in cell elongation, in part by controlling a plethora of cell wall biosynthesis and remodelling genes (Sun et al., 2010, Belkhadir and Jaillais, 2015, Singh and Savaldi-Goldstein, 2015). We recently revealed that when cell wall integrity is challenged by interference with the activity of the important cell wall modification enzyme pectin methylesterase (PME), BR signalling is activated as a compensatory mechanism (Wolf et al., 2012b). This BR-mediated compensatory response depends on RECEPTOR-LIKE PROTEIN 44 (RLP44) (Wolf et al., 2014). RLP44 directly 
interacts with both BRI1 and BAK1 and promotes their association (Holzwart et al., 2018b). However, RLP44 also interacts with and promotes the activity of the receptor complex for the plant growth peptide phytosulfokine (PSK) (Sauter, 2015, Holzwart et al., 2018b). The interaction between RLP44 and the PSK receptor PSKR1 is important for the maintenance of procambial cell fate, as both RLP44 and PSK-related mutants show ectopic xylem formation in the position of the procambium in seedling roots (Holzwart et al., 2018b). Thus, RLP44 acts in at least two different LRR-RLK pathways through direct interaction with their receptors. Multi-faceted interactions among LRR proteins is an emerging theme in plant receptor biology (Ma et al., 2016, Smakowska-Luzan et al., 2018); however, it is not clear how distinct responses are ensured. As RLP44 acts in two separate pathways with well-defined read-outs, it provides an excellent model to decipher how pathway specificity is achieved. Here, we show that RLP44 is phosphorylated in its highly conserved C-terminal cytosolic tail. This posttranslational modification is crucial for regulating RLP44's interaction with BRI1 and thus its function in BR signalling activation. RLP44 variants in which phosphorylation is blocked enter endocytosis prematurely, leading to an almost entirely intracellular localization. Conversely, mimicking phosphorylation results in preferential RLP44 localization at the plasma membrane. This increase in the ratio of plasma membrane to intracellular localization is dependent on the presence of BRI1, suggesting that phosphorylation affects subcellular localization through modulating the interactions of the LRR proteins. In contrast, the interaction with PSKR1 is not affected by RLP44 phospho-status. Analysis of the contribution of individual amino acid modifications suggests that association of RLP44 with its target receptor complexes is controlled by its phosphorylation pattern, providing a framework to understand how specificity can be determined in plasma membrane receptor complex interactions.

\section{Results}

\section{Four conserved putative phosphorylation sites are required for RLP44-mediated BR signalling activation}

AtRLP44 is unusual compared to other RLPs in Arabidopsis, as its juxtamembrane domain is not acidic, and its cytoplasmic, C-terminal tail shows a pl of 4.7, whereas the majority of Arabidopsis RLPS harbour cytoplasmic tails with quite basic pl (Gust and Felix, 2014). However, this unusual cytoplasmic domain (CD) is well conserved among the apparent RLP44 orthologues in land plants (Fig. 1A and Fig. S1). Interestingly, of four putative phosphorylation sites in AtRLP44 (from hereon RLP44), three - T256, S268, and Y274 - are conserved in all orthologues, whereas S270 seems to be specific to Brassicacea. All four sites are predicted to be phosphorylated (NetPhos 3.1 server (www.cbs.dtu.dk/services/NetPhos/)) and we have previously obtained evidence for serine phosphorylation in RLP44 using anti-phosphoserine antibodies (Wolf et al., 2014). To assess which of the four amino acids are phosphorylated in vivo, we performed mass spectroscopy after immunoprecipitation from transgenic Arabidopsis thaliana plants expressing an RLP44-GFP fusion protein, as well as from transiently transformed Nicotiana benthamiana leaves. Peptide coverage was quite poor, in particular from Arabidopsis, despite effective immunopurification of RLP44 fusion protein. However, we were able to identify S268 phosphorylation in N. benthamiana (Fig. S2). As we could not rule out modification of the other three residues, we first assessed the effect of blocking posttranslational modification of all four putative phospho-sites. To this end, we generated a version of RLP44 fused to GFP in which all four sites are mutated to either alanine (T256A, S268A, A270A) or phenylalanine (Y274F) and termed this phospho-dead variant RLP44-GFP Pdead. Conversely, we created a phospho-mimic (Pmimic) version of RLP44-GFP in which all four putative phospho-sites are converted to Glutamate (T256E, S286E, A270E, Y274E). Throughout the manuscript, we refer to these genetic modifications of putative 
phosphorylation sites as affecting "phospho-status" for brevity. Constructs encoding these mutant versions of RLP44-GFP along with a wild-type version (RLP44-GFP WT) were used to transform the rlp44 mutant cnu2 (Wolf et al., 2014) for complementation assays. We originally described cnu2 as a suppressor mutant of an overexpression line of PMEI5 (PMElox), which displays growth defects due to a compensatory boosting of brassinosteroid signalling strength. Activation of BR signalling in response to PMEI-mediated reduction of PME activity critically depends on RLP44, which directly interacts with the BR receptor BRI1 and its co-receptor BAK1. Mutation of RLP44 in cnu2 thus leads to relatively normal growth despite the presence of the PMElox transgene. As previously described (Wolf et al., 2014), expression of RLP44-GFP is able to complement cnu2 and results in recovery of the PMElox seedling root waving phenotype in several independent transgenic lines (Fig. 1D and Fig. S3A). Similarly, the Pmimic variant of RLP44-GFP could restore or even slightly enhance the PMElox phenotype, suggesting that the presence of the native version of the four mutated RLP44 sites is not essential for function in BR signalling activation and that mimicking phosphorylation at these sites might be associated with enhanced activity. In contrast, the Pdead version consistently failed to complement cnu2 with respect to the root waving phenotype (Fig 1D and Fig. S3A). Concentrating on one line for each construct with comparable RLP44-GFP expression levels (Fig. S3B), we made similar observations for other previously described PMElox phenotypes (Wolf et al., 2012b), such as altered expression of BR marker genes (Fig. S3C, D), reduced seed yield (Fig. S3E), and agravitropic growth on vertical agar plates in the dark due to enhanced BR signalling (Fig. S4). In each case, the line expressing the WT or Pmimic versions of RLP44-GFP in the cnu2 suppressor mutant behaved like PMElox, whereas the line expressing the Pdead version behaved like cnu2, i.e. similar to wild type. We then assessed the ability of the three RLP44-GFP versions to rescue r/p44 phenotypes in the absence of cell wall challenge induced by the PMElox transgene. Mutants of RLP44 such as rlp44 ${ }^{\text {cnu2 }}$ show reduced petiole length (Wolf et al., 2014), presumably caused by impaired BR signalling. Expression of RLP44-GFP WT and RLP4-GFP Pmimic, but not RLP44-GFP Pdead, could restore the petiole length defect of $r l p 44^{\text {cnu2 }}$ (Fig. S5), in line with the assumption that this phenotype is BR signalling-related. In conclusion, blocking phosphorylation of four putative phosphosites in the cytoplasmic domain precluded BR signalling activation-related functions of RLP44. In contrast, introducing a negative charge to mimic phosphorylation at these sites resulted in functionality comparable to that of the wildtype version of RLP44-GFP.

\section{Phospho-status affects the subcellular localization of RLP44}

We have previously shown that RLP44-GFP is localized in intracellular vesicles and at the plasma membrane (Wolf et al., 2014), in agreement with its association with receptors for extracellular signalling ligands. To assess whether modification of the four putative phosphorylation sites influences the subcellular localization of RLP44-GFP, we imaged root tips of transgenic lines expressing RLP44-GFP WT, Pdead or Pmimic in the wild-type (Col-0) background. RLP44-GFP showed the expected distribution of plasma membrane and intracellular fluorescence, which partially co-localized with the styryl dye FM4-64 (Fig. 2A). Surprisingly, RLP44-GFP Pdead showed only very faint plasma membrane fluorescence and almost exclusive labelling of intracellular vesicles that appeared to largely co-localize with FM4-64 30 minutes after its application, suggesting endosomal localization (Fig. 2A). In sharp contrast, RLP44-GFP Pmimic showed strongly enhanced plasma memebrane labelling with only few intracellular vesicles (Fig. 2A). Similar results were observed in the cnu2 background (Fig. 2B) and confirmed by quantification of the mean plasma membrane to intracellular fluorescence ratio (Fig. 2C). These results would be consistent with two different and mutually exclusive scenarios: first, the putative phosphorylation sites might be required for export of RLP44-GFP, thus the Pdead fluorescence distribution might reflect a failure of the receptor to 
be transported to the plasma membrane, whereas mimicking phosphorylation in Pmimic might enhance transport to the surface; second, differential rates of endocytic uptake might underlie the contrasting behaviour of the Pdead and Pmimic versions of RLP44-GFP. In the latter scenario, Pdead would be transported to the plasma membrane initially, but would rapidly undergo endocytosis, whereas mimicking phosphorylation would block internalization. As the second scenario is favoured by an increased Pdead fluorescence in expanding and fusing cell plates (Fig. 2C), which exhibit rearrangement of cellular trafficking towards secretion (Richter et al., 2014), we sought to test the hypothesis that the three RLP44-GFP versions differ in their rate of endocytosis. To this end, we first interfered with endosomal trafficking by applying the phosphoinositide 3-kinase inhibitor Wortmannin $(\mathrm{Wm})$. This treatment leads to a swelling of the multi-vesicular bodies (MVBs)/late endosomes (LE), thus indicating late endosomal nature of sensitive structures (Wang et al., 2009, Viotti et al., 2010). In addition, Wm can indirectly lead to an inhibition of endocytosis (Emans et al., 2002). All three RLP44-GFP version were sensitive to Wm, as subpopulations of intracellular GFP-positive punctate showed pronounced swelling, suggesting that RLP44-GFP reaches late endosomes. Moreover, plasma membrane labelling of RLP44-GFP Pdead was increased after Wm treatment, consistent with the hypothesis that this RLP44 version displays low steady state abundance at the plasma membrane because it experiences increased endocytic uptake, thus inhibition of this uptake, in this case by $\mathrm{Wm}$, leads to an increase in abundance at the plasma membrane. To independently corroborate these results, we made use of the fungal toxin brefeldin A (BFA), which, in Arabidopsis roots, leads to aggregation of endosomal compartments into a hybrid organelle, the BFA compartment, in which endocytic cargo becomes trapped (Geldner et al., 2003, Grebe et al., 2003, Dettmer et al., 2006, Viotti et al., 2010). Consequently, quantification of fusion protein-derived fluorescence in BFA compartments has been used to assess endocytosis of plasma membrane receptors (Di Rubbo et al., 2013, Martins et al., 2015). After 120 minutes of BFA treatment, the three RLP44-GFP versions showed differential accumulation in BFA compartments, with the Pdead version showing the strongest signal, followed by WT and Pmimic. These observations are consistent with differential endocytosis as the mechanistic explanation for the different subcellular distribution of RLP44-GFP WT, Pdead, and Pmimic. In order to directly test the impact of retrograde trafficking on RLP44-GFP localization through genetic interference with endocytic uptake, we used a previously described line expressing, in an inducible manner, artificial miRNAs directed against the TPLATE adapter complex to block clathrin-mediated endocytosis (Van Damme et al., 2011, Gadeyne et al., 2014). As expected, induction of amiRNA expression for 48 hours led to a marked increase in the ratio of plasma membrane -localized to intracellular FM4-64 fluorescence and a dramatic reduction in size and quantity of FM4-64-positive BFA compartments (Fig. 4A, B), indicating strongly decreased endocytosis. Importantly, amiRNA induction in plants expressing RLP44-GFP Pdead led to the appearance of GFP fluorescence at the plasma membrane, in sharp contrast to mock treatment (Fig. 5A, B). Quantification of plasma membrane and intracellular GFP fluorescence in RLP44-GFP WT and Pdead lines revealed that inhibition of clathrin-mediated endocytosis leads to a WT-like fluorescence distribution of RLP44-GFP Pdead (Fig. 4B), suggesting that phospho-status governs endosomal trafficking of RLP44.

\section{RLP44 phosphorylation is required for its interaction with BRI1}

To assess whether the inability of the RLP44-GFP Pdead version to activate BR signalling was due to an altered behaviour in protein-protein interactions, we performed a ratiometric Bimolecular Fluorescence Complementation (BiFC) assay after transient expression in $N$. benthamiana (Grefen and Blatt, 2012). To this end, we fused RLP44-GFP WT, Pdead, and Pmimic to the C-terminal part of YFP, whereas BAK1 and BRI1 were fused to the YFP Nterminus. Quantification of reconstituted YFP fluorescence and normalization to RFP 
expressed constitutively from the same transgene revealed that all three RLP44 versions were able to interact with BAK1 (Fig. 5), as previously shown for the WT version (Wolf et al., 2014). The WT and Pmimic version also yielded YFP fluorescence when co-expressed with BRI1 fused to N-YFP. However, RLP44 Pdead did not show interaction with BRI1, as fluorescence levels were not increased compared to the combination with the related LRR-RLK FLAGELLIN SENSING2 (FLS2), serving as negative control (Fig. 5). These results are unlikely to be caused by the different trafficking dynamics of RLP44-GFP Pdead, as i) this RLP44 variant was able to interact with BAK1, ii) reconstituted fluorescent protein in BiFC assays locks the interaction partners in a permanent complex (Cabantous et al., 2005, Magliery et al., 2005), and iii) the differences in subcellular localization between the three RLP44-GFP variants are less pronounced after transient expression in $N$. benthamiana (Fig S6). To summarize, blocking post-translational modification in the Pdead mutant rendered RLP44 unable to interact with BRI1, suggesting that phosphorylation is required for RLP44's role in promoting $\mathrm{BR}$ signalling at the BR receptor level.

\section{Subcellular distribution of RLP44 is modified by BRI1}

To summarize our results so far, we have shown that blocking post-translational modification of four putative phosphorylation sites in RLP44 impedes its function in BR signalling, interferes with its ability to interact with BRI1, and promotes its accumulation in endosomes at the expense of plasma membrane localization. Together, these observations would be consistent with a model in which biochemical interaction with the RLK would determine subcellular localization. To test this hypothesis, we quantified the subcellular distribution of RLP44 fluorescence in the absence of BRI1. To this end, we crossed RLP44-GFP WT, Pdead, and Pmimic into the T-DNA insertion line bri1-null (Jaillais et al., 2011a), which, based on the available evidence, completely lacks BRI1. Interestingly, plasma membrane localization of the RLP44-GFP WT version was slightly enhanced in the bri1-null mutant, whereas the distribution of fluorescence derived from RLP44-GFP Pdead was not strongly affected (Fig. 6A, B). This suggests that interaction with BRI1 affects subcellular localization, although the increase in plasma membrane localization of the WT version is difficult to interpret given that thousands of genes are differentially regulated in bri1 null mutants (Chaiwanon and Wang, 2015) and that the absence of BRI1 likely affects the composition of other RLK complexes in the plasma membrane. Strikingly, the almost exclusive plasma membrane localization of RLP44-GFP Pmimic clearly depended on the presence of BRI1, as intracellular labelling was strongly increased in bri1-null compared to the Col-0 background (Fig. 6A) and the plasma membrane -to-cytosol fluorescence ratio of Pmimc was not increased compared to the RLP44-GFP WT in the mutant background (Fig. 6B). These results suggest that, at least in part, interaction with BRI1 precludes endocytosis of the Pmimic version of RLP44. Accordingly, the behaviour of the Pdead variant, which is unable to interact with BRI1 (Fig. 5), is unaffected by the presence of BRI1.

\section{Phosphorylation at individual sites has opposing effects on RLP44 function}

To decipher the contribution of individual putative phosphorylation sites to RLP44 function, we mutated each of the sites individually to alanine in RLP44-GFP and quantified the capacity of these constructs to complement cnu2. We reasoned that working with near endogenous expression levels might increase the sensitivity of the complementation assay, therefore all RLP44-GFP variants were driven by the RLP44 promoter (Holzwart et al., 2018b). To assess comparability between the previously characterized overexpression lines, we also generated RLP44 promoter-driven Pdead and Pmimic versions, in which all four putative phosphosites are mutated as previously described. After transformation and selection of transgenic plants, we categorized the phenotype of individual T1 transformants as non-complemented (cnu2like) or complemented (PMElox-like). The pRLP44:RLP44-GFP WT construct was able to complement cnu2 in about half of the transformants, demonstrating that the fusion protein was 
functional (Fig. 7A). In line with previous results, the $p R L P 44: R L P 44-G F P$ Pmimic construct appeared to be slightly more potent, whereas the Pdead version was significantly reduced in its capacity to complement cnu2 (Fig. 7A). Furthermore, Confocal laser scanning microscopy (CLSM) analysis revealed that the subcellular distribution of RLP44-GFP-derived fluorescence of the overexpression lines was recapitulated in the corresponding endogenous promoter lines (Fig. 7B, C). Interestingly, constructs mutated in single amino acids strongly differed from each other in their ability to complement cnu2. None of the pRLP44:RLP44-GFP T256A transformants showed PMElox phenotype. However, we were not able to demonstrate accumulation of GFP fluorescence, suggesting that this particular mutation might affect protein stability. Strikingly, the pRLP44:RLP44-GFP S268A construct yielded the highest proportion of complemented T1 plants of all versions tested, including WT and Pmimic, and showed almost exclusively plasma membrane localization, whereas the S270A and Y274F variants were unable to complement cnu2 and showed intracellular localization (Fig. 7A). This suggests that S268 and S270/Y274 have opposing functions and that phosphorylation at S268 has a strong negative effect on BR signalling activation. Consistent with this notion, the Pdead and Pmimic variants appear to display a combinatorial effect of mutating the antagonistic residues based on the rate of complementation (Fig. 7A). Our results are consistent with a model in which phosphorylation of the cytoplasmic RLP44 tail determines whether RLP44 functions in BR signalling with a strong negative effect of phosphorylation at S268 and opposite effects of some or all of the other three putative phospho-sites.

\section{Phosphorylation is not required for the role of RLP44in PSK signalling}

We have recently described (Holzwart et al., 2018a) that, apart from promoting BR signalling, RLP44 plays a role in promoting signalling mediated by the receptors of PSK peptide hormones, PSKR1 and PSKR2 (Matsubayashi et al., 2002, Matsubayashi et al., 2006). Having demonstrated that the role of RLP44 in BR signalling depends on the four phosphosites in the cytosolic tail, we next assessed whether the Pdead and Pmimic versions affected functionality of RLP44 in the PSK pathway. To this end, we analysed PSK-related phenotypes in complemented $r / p 44^{\text {cnu2 }}$ mutants. PSK signalling is required in the epidermis for normal root elongation (Kutschmar et al., 2009, Hartmann et al., 2013) and exogenously applied PSK peptide leads to a moderate increase in the root length of wild type seedlings (Kutschmar et al., 2009, Ladwig et al., 2015, Wang et al., 2015a). In line with its role in promoting PSK signalling, rlp44 ${ }^{\text {cnu2 } 2}$ is compromised in this response (Holzwart et al., 2018b). Analysis of the complementation lines described earlier (Fig. S5) revealed that all three RLP44-GFP versions, including Pdead, were able to restore the response to PSK in rlp44 ${ }^{\text {cnu2 }}$ (Fig. 8A), suggesting that PSK and BR signalling might have different requirements for the modification of the four RLP44 phosphorylation sites. Notably, the PSK signalling-mediated promotion of root growth is believed to occur in the epidermis, a tissue where RLP44, BRI1 and PSKR1 are co-expressed (Friedrichsen et al., 2000, Matsubayashi et al., 2006, Kutschmar et al., 2009, Holzwart et al., 2018b). To corroborate these results, we tested whether the Pdead version could complement the ectopic xylem phenotype of rlp44 ${ }^{\text {cnu2 }}$ seedling roots, which is caused by reduced PSK signalling (Holzwart et al., 2018b). In line with the root length assay, expression of RLP44-GFP Pdead led to wild type-like xylem cell numbers (Fig. 8B), indicating that, indeed, phosphorylation of these residues is not a requirement for the role of RLP44 in promoting PSK signalling. To exclude a PSK signalling-independent effect of RLP44 Pdead on xylem cell numbers we performed a rescue experiment in pskr1-3 pskr2-1 double mutants. As previously described for the WT RLP44-GFP (Holzwart et al., 2018b), RLP44 Pdead requires the presence of PSK receptors to exert any effect on xylem cell number (Fig. $8 \mathrm{C})$. Finally, in line with the hypothesis that RLP44 phosphorylation is not required to promote PSK signalling, all three RLP44 versions were able to interact with PSKR1 in ratiometric BiFC assays after transient expression in $N$. benthamiana (Fig. 9E), in contrast to what was 
previously observed for the interaction with BRI1 (Fig. 5). In summary, our study suggests that phosphorylation determines RLP44 functionality in different receptor complexes and demonstrates how selectivity between different receptor pathways can be encoded in the pattern of post-translational modifications.

\section{Discussion}

Plants rely on a complex network of cell surface receptors to integrate developmental and environmental cues into behaviour adapted to the conditions. In light of the many possible interactions between LRR-RLKs (Smakowska-Luzan et al., 2018) and the high likelihood of accidental encounters in the crowded plasma membrane, which restricts mobility to two dimensions, a central question in signal transduction is thus how a specific response that transcends noise is ensured. In the current model of LRR-RLK signalling activation, the extracellular ligands serve as molecular tether to bring receptor and co-receptor together and thereby juxtapose their kinase domains in the cytosol, so that trans-phosphorylation can ensue (Hohmann et al., 2017). Fully activated kinase domains then recruit downstream signal transduction components which can be dedicated to individual signalling pathways (He et al., 2000, Brutus et al., 2010, Hohmann et al., 2018). For example, a chimeric receptor constituted by the BRI1 extracellular domain and the cytosolic domain of the immune receptor XA21 triggered immune signalling in response to BRs (He et al., 2000), whereas a similar BRI1HAESA chimera was able to complement haesa mutants in a BR dependent manner (Hohmann et al., 2018). However, several pathways share components, as demonstrated by the ubiquity of SERK co-receptors (Ma et al., 2016), which are also an essential component of both PSK and BR signalling (Ladwig et al., 2015, Wang et al., 2015a), the two LRR-RLKgoverned pathways studied here. In addition to RLKs, RLPs contribute to receptor-mediated signalling and increase the complexity of the plasma membrane receptor network. We have previously demonstrated that RLP44 can promote both PSKR1 and BRI1-mediated signalling, but it was unclear how these activities are coordinated in light of the fact that RLP44 seems to act independently of extracellular ligands (Wolf et al., 2014, Holzwart et al., 2018b). Here, we show that phospho-status can route RLP44s towards functioning in PSK or BR signalling. Individual residues seem to play diverging roles, as indicated by their opposite effect on physiological read-outs of RLP44 function. This is consistent with previous proteomic analysis that revealed that putative phosphorylation sites are enriched in the binding interfaces of heterooligomers (Nishi et al., 2011) and proteins that engage in multiple (mutually exclusive) interactions using the same contact surface, presumable acting as a switch between different pathways (Tyagi et al., 2009). While we could not unequivocally demonstrate phosphorylation of T256, S270, and Y274 or completely resolve the individual contribution of these residues, their modification generally seems to promote interaction with BRI1 and function in the BRI1mediated signalling pathway. In contrast, phosphorylation of S268 seems to exert a negative effect on RLP44 function in BR signalling. This idea is based on the observation that the S268A mutant had the highest capacity for complementing BRI1-related rlp44 mutant phenotypes, exceeding both wild type and phosphomimic variants. In addition, rendering all four phosphosites simultaneously unable to be phosphorylated did not completely block BR-mediated RLP44 function, in contrast to individual phospho-dead version of residues other than S268. Notably, this latter observation could be explained by two different mechanisms. Phosphorylation of these residues might be required for the interaction with BRI1 or, alternatively, inhibit phosphorylation of S268, consistent with frequent observation of crosstalk between clustered posttranslational modification sites (Hunter, 2007, Venne et al., 2014). More work will be needed to decipher these potential interdependencies among residues in the cytoplasmic domain of RLP44 and identify the enzymes involved in their modification. 
Notably, the activity of BRI1 itself has been linked to controlling the interaction with regulatory proteins. BRI1 phosphorylates its inhibitory interaction partners BKI1 and BIK1 to trigger their release from the receptor complex (Jaillais et al., 2011b, Lin et al., 2013). It is thus conceivable that S268 phosphorylation, by BRI1 or another kinase, serves to temporally limit the RLP44mediated promotion of BR signalling.

Post translational modifications play a central role in regulating the trafficking of plasma membrane proteins. It is well established that ubiquitination can act as a signal for internalization and endosomal sorting across kingdoms (Haglund and Dikic, 2012, Dubeaux et al., 2015). The 76 amino acid protein ubiquitin is linked via its C-terminal glycine to lysine residues in the target protein. Endosomal trafficking of the LRR-RLKs FLS2 and BRI1 has been shown to depend on ubiquitination (Lu et al., 2011, Martins et al., 2015, Zhou et al., 2018). Several prominent examples from the animal literature demonstrate the versatility of phosphorylation for the regulation of trafficking (Offringa and Huang, 2013). For instance, phosphorylation of epidermal growth factor receptor (EGFR) triggers interaction with the AP2 adaptor complex and a E3 ubiquitin ligase, respectively, to promote endocytosis through two different pathways (Bakker et al., 2017). In plants, only for very few plasma membrane receptor proteins a direct link between phosphorylation and an effect on trafficking has been demonstrated. Endocytosis of LYSIN MOTIF-CONTAINING RECEPTOR-LIKE KINASE5 (LYK5) appears to be regulated by CHITIN ELICITOR RECEPTOR KINASE 1 (CERK1)mediated phosphorylation (Erwig et al., 2017). In addition, a phospho-dead mutant of FLS2 (T867A) shows reduced endocytosis, and the same effect is observed when kinase activity is pharmacologically inhibited (Robatzek et al., 2006); however, ubiquitination is not dependent on phosphorylation of this residue (Lu et al., 2011). Besides FLS2, many LRR-RLKs undergo ligand binding-induced endocytosis. While a direct regulatory role of specific phosphorylation events, for example through mediating interaction with the ubiquitination machinery, has yet to be shown, receptor complex auto- and trans-phosphorylation is the primary output of ligand perception and thus likely to be involved in coupling receptor activation to endocytosis. Interestingly, RLP44 shows a highly conserved lysine at position 266 . This is in close proximity to the conserved and phosphorylated residue S268, which we have shown here to strongly impact the subcellular localization of RLP44. It is thus tempting to speculate that phosphorylation might affect ubiquitination (Swaney et al., 2013, Venne et al., 2014), as previously observed with plasma membrane proteins such as EGFR (Sigismund et al., 2013). However, in our mass spectrometry data, we find the characteristic ubiquitin di-glycine remnant (after trypsin digestion) on Lys266 of both RLP44-GFP WT and RLP44-GFP Pdead (Fig. S2C, E). Although these data do not rule out an impact of phosphorylation on the quantity or type of ubiquitination, they do show that phosphorylation is not required for the decoration with ubiquitin per se. Our results thus point to a slightly different scenario: RLP44 might be constitutively primed for endocytosis, and its dwell time at the membrane might be determined by the interactions it engages in. In favour of this hypothesis, endosomal uptake of RLP44GFP Pmimic was strongly enhanced in the absence of BRI1. Further supporting this scenario are our BiFC results, which showed inability of Pdead RLP44 to interact with BRI1, whereas interaction with PSKR1 was not affected. As it is assumed that the reconstituted fluorescent protein in BiFC assays locks the interaction partners in a permanent complex (Cabantous et al., 2005, Magliery et al., 2005), these results should be independent of trafficking dynamics after the proteins had the chance to interact, for example upon delivery to the plasma membrane. Supporting this view, all RLP44 variants, including Pdead, showed similar BiFC interaction with the plasma membrane-localized BAK1. Thus, phospho-status acts as a biochemical determinant for RLP-RLK interactions rather than as cue for the trafficking machinery. Taken together, our findings also suggest that RLP44 and BRI1 don't necessarily enter endocytosis as part of one complex. Notably, RLP44 phospho-status had no impact on its interaction with PSKR1 or its functionality in the PSK pathway, despite RLP44-GFP Pdead 
showing very low abundance at the plasma membrane. This raises the question of whether PSKR1 signalling can occur from endosomes, as it is assumed for diverse signalling pathways in animals (Howe and Mobley, 2004, Sigismund et al., 2012) and also discussed in plants (Geldner and Jurgens, 2006). Alternatively, brief presence of RLP44 Pdead at the plasma membrane might be sufficient for supporting PSK signalling. To assess whether membrane sub-compartmentalization contributes to the maintenance of specificity, it will be important to track trafficking of RLP44 together with its interaction partners BRI1 and PSKR1 using advanced imaging technology (Bucherl et al., 2017).

We found apparent orthologues of RLP44 in all analysed plant species, suggesting that RLP44-like genes are under strong selective pressure, similar to other RLPs involved in development such as CLV2 and TMM (Fritz-Laylin et al., 2005). Notably, T256, S268, Y274 are strictly conserved in all RLP44 orthologues, despite the tendency of phosphorylation sites to diverge rapidly in linear motifs and receptor proteins (Holt et al., 2009, Riano-Pachon et al., 2010, Beltrao et al., 2012). In contrast, S270 is only found in Brassicacea and did not substitute an acidic residue, which is assumed to contribute a dynamic switch to a pre-existing interaction (Pearlman et al., 2011). In this respect, it is noteworthy that we identified RLP44 as required in Arabidopsis for the response to changes in pectin de-methylesterification (Wolf et al., 2014). This cell wall modification apparently evolved as an ancient cell wall consolidation mechanism and is operative in extant members of the charophycean green algae, which are the closest living relatives of land plants (Proseus and Boyer, 2006, Popper et al., 2011, Wolf et al., 2012a, Domozych et al., 2014, Nishiyama et al., 2018). On the other hand, it has been questioned whether BRI1-like brassinosteroid receptors are present in ancestral vascular plants (Cheon et al., 2013), thus RLP44 orthologues could predate some of its interaction partners. It will be interesting to dissect how a protein like RLP44, which modulates the function of distinct RLKs, co-evolved with its interaction partners.

\section{Experimental Procedures}

\section{Plant material and growth conditions}

All plants used in this study were of the Col-0 ecotype and are described in Table S1. Seeds were sterilized with $1.2 \% \mathrm{NaOCl}$ in $70 \%$ ethanol and washed twice with absolute ethanol, and dried under sterile conditions. Plants were grown in $1 / 2$ strength MS medium supplemented with $1 \%$ sucrose and $0.9 \%$ plant agar.

\section{Cloning}

35S:RLP44-GFP was generated by amplifying the coding sequence of the (intronless) $R L P 44$ from genomic DNA using primers SW660 and SW670, and subsequent Gateway cloning into pDONR207 and pK7FWG2 (Karimi et al., 2002). RLP44-GFP Pdead was generated by introducing the T254A mutation with site-directed mutagenesis using primers SW666 and SW667 and RLP44 in pDONR207 as template. After recombination reaction into pK7FWG2, this plasmid was used as a PCR template to introduce the remaining three mutations with primers SW668 and SW660. The resulting PCR product was introduced in pDNOR207 through BP reaction, after which a sequence-confirmed clone was used for $L R$ reaction into pK7FWG2. RLP44-GFP Pmimic was created analogously using primers SW672 and SW673 to introduce the T256E mutation and primers SW671 and SW660 to introduce the remaining mutations via PCR. The resulting PCR product was introduced in pDNOR207 through BP reaction, after which a sequence-confirmed clone was used for LR reaction into pK7FWG2. Similarly, constructs containing individual mutations S268A (SW661), S270A (SW662), and Y274F (SW663), were generated using primer SW660 and the indicated reverse primers and used as PCR template for the generation of pRLP44-RLP44-GFP constructs through 
GreenGate (Lampropoulos et al., 2013) cloning (Table S3). Ratiometric split YFP constructs were generated through pDONR221 P2P3 entry modules for fusions to the N-terminal part of YFP and pDONR221 P1P4 entry modules for fusions to the C-terminal part of YFP using the primers listed in Table S2 and Gateway BP reaction according to the manufacturer's instructions. Sequence-confirmed clones were used for $L R$ reactions into the pBiFCT 2in1-CC vector (Grefen and Blatt, 2012). All other constructs are based on the GreenGate system (Lampropoulos et al., 2013); for details, see Table S3.

\section{Transient expression in $\mathbf{N}$. benthamiana}

For Agroinfiltration of $N$. benthamiana leaves, Agrobacterium tumefaciens strains C58C1 (for Gateway constructs) or ASE (For Greengate constructs) were used. As pre-culture, single Agrobacterium colonies were used to inoculate $5 \mathrm{~mL}$ of LB medium supplied with the corresponding antibiotics overnight at $28{ }^{\circ} \mathrm{C}$ with agitation (200 rpm). The main culture was started by transferring $100 \mu \mathrm{l}$ of the pre culture to $30 \mathrm{~mL}$ of LB medium supplied with the corresponding antibiotics. The main culture was grown overnight at $28{ }^{\circ} \mathrm{C}$ with shaking $(200$ rpm). Thereafter, the Agrobacteria culture was centrifuged for $30 \mathrm{~min}$ at $3000 \times \mathrm{g}$, the supernatant discarded and the pellet resuspended in $10 \mathrm{~mL}$ of distilled water. Resuspended culture was starved for one hour at ambient temperature under moderate agitation (100 rpm). Agrobacteria concentration was adjusted to $\mathrm{OD}_{600}=0.5$. Two-to-three week-old $N$. benthamiana plants were grown under long day conditions ( $16 \mathrm{~h}$ photoperiod, $100 \mu \mathrm{E} \mathrm{m-2} \mathrm{s-}$ 1 light intensity, $65 \%$ humidity and $25^{\circ} \mathrm{C}$ ). Three hours before agroinfiltration, plants were moved to lab light and kept under humid conditions. Infiltration was performed on the abaxial side of $N$. benthamiana leaves using a $1.5 \mathrm{~mL}$ needleless syringe. After infiltration, plants were kept in a humid and light-protected environment overnight and transferred to long day conditions afterwards. Two days after infiltration, leaf discs were used for confocal microscope observation of fluorescent signal and BiFC or several leaves were collected and frozen in liquid nitrogen for subsequent protein extraction.

\section{Immunopurification and mass spectroscopy analysis}

RLP44-GFP (WT or mutant variants) were transiently expressed in three- to four-week-old $N$. benthamiana leaves by agroinfiltration as previously described. Samples were taken two days post-infiltration; accumulation of the GFP-fused proteins was confirmed by confocal microscopy. Plant tissue was ground in liquid nitrogen, total proteins were extracted by adding lysis buffer (100 mM Tris- $\mathrm{HCl} \mathrm{pH} 8.0 ; 150 \mathrm{mM} \mathrm{NaCl}$; 10\% glycerol; 5 mM EDTA; 5mM DTT, $1 \mathrm{mM}$ PMSF; $1 \%$ protease inhibitor cocktail; $1 \%$ NP-40) and the extracts were cleaned by filtration; extracts were incubated with GFP-Trap beads (Chromotek, Germany) for one hour, and beads were subsequently washed using washing buffer with detergent (100 mM Tris-HCL pH 8.0; $150 \mathrm{mM} \mathrm{NaCl} ; 10 \%$ glycerol; $2 \mathrm{mM}$ DTT; $1 \%$ protease inhibitor cocktail; $0.2 \% \mathrm{NP}-40$ ) three times and washing buffer without detergent $(100 \mathrm{mM}$ Tris-HCL pH 8.0; $150 \mathrm{mM} \mathrm{NaCl}$; $10 \%$ glycerol; 2mM DTT; $1 \%$ protease inhibitor cocktail) twice.

Mass spectrometry analysis was performed at the Proteomics Core Facility of the Shanghai Center for Plant Stress Biology. Matching raw MS data to peptide sequences was performed using Mascot software with the annotated proteins from the $N$. benthamiana draft genome sequence v. 0.4.4, which was obtained from the International Solanaceae Genomics Project (SOL) (https://solgenomics.net/), and AtRLP44-GFP sequence.

\section{Quantitative Real-Time PCR}

For RNA analysis, a maximum of $100 \mathrm{mg}$ of frozen $A$. thaliana seedling material was ground in a $2 \mathrm{ml}$ reaction tube with the aid of a pre-cooled tissue lyser (Tissuelyserll, Qiagen). RNA from ground tissue was extracted with an RNA purification Kit (Roboklon), following the manufacturer's instructions. Synthesis of cDNA was carried out using AMV Reverse 
Trascriptase (EURx) following the manufacturer's instructions. The cDNA reaction was diluted 1:10 in water and used for qPCR analysis with primers directed against BR marker genes EXPA8 and DWF4. The SYBRR Green I nucleic acid gel stain (Sigma-Aldrich) was used for amplification, CLATHRIN (At1g10730) was used as reference gene. qPCR reactions were run in a Rotor-Gene Q 2plex (Qiagen) and the amplification data analyzed by the 75 Rotor-Gene Q 2plex software. For primers, see Table S2.

\section{Genotyping}

Presence of the rlp44 ${ }^{\text {cnu2 }}$ mutation was assessed by CAPS marker using primers SW503 and SW504 and subsequent Hinfl digestion. For the genotyping of the bri1-null T-DNA insertion, primers SW1378 and SW1379 were used for detection of the wild type allele. Presence of the T-DNA insertion was assessed with primers SW1377 and SW1379. For genotyping pskr1-3, primers SW1745 and SW1746 were used to assess presence of the wild type allele, and SW130 and SW1746 to assess presence of the T-DNA insertion. Primers SW1984 and SW1985 were used to detect the presence of the PSKR2 wild type allele, and SW230 and SW1985 were used to detect the T-DNA. Presence of Gateway insertions was checked by using primers SW905 and SW906 directed against the attB1 and attB2 sequences, respectively. Presence of GreenGate insertions was assessed by PCR with primers SW1202 and SW1137.

\section{Bimolecular Fluorescence Complementation}

Ratiometric split YFP using the Gateway destination vector pBiFCT 2in1-CC was carried out as described (Grefen and Blatt, 2012). Briefly, N-terminal fusions to the C-terminal or Nterminal part of YFP were assembled in pBiFCT 2in1-CC (see Table S2), which also carries a 35S:RFP cassette for normalization. To test interaction, the different pBiFCT 2in1-CC constructs were mobilized in Agrobacteria, which were then used to infiltrate $N$. benthamiana leaves. Two discs per leaf were analysed by CLSM for YFP and RFP signal using four images from different regions. At least three different leaves in 3 independent experiments were used for the image analysis.

\section{Xylem cell number analysis}

Basic fuchsin staining of five-day-old seedling roots and CLSM analysis was performed as described (Holzwart et al., 2018b).

\section{Microscopy}

CLSM of Arabidopsis roots and N. benthamiana leaf discs was performed on a TCS SP5 II inverted Confocal Laser Scanning Microscope (Leica) or a LSM 510 Meta Confocal Laser Scanning Microscope (Zeiss). In the first case, a HCX PL APO lambda blue 63.0x1.20 water immersion objective (Leica) was used. In the second case, a Plan-Neofluoar 5.0x1.05, Plan-

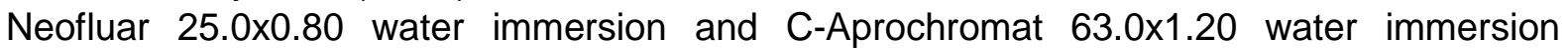
objectives were used. Excitation wavelength was set to $488 \mathrm{~nm}$ for GFP, 514 for YFP, and $561 \mathrm{~nm}$ for RFP or mCherry. Emission was recorded at $500-545 \mathrm{~nm}$ for GFP, at $545-573 \mathrm{~nm}$ for YFP and 620-670 nm for RFP or mCherry using HyD hybrid detectors (Leica) or photomultipliers (PMT) detectors (Zeiss). For inhibitor treatments, 6-day-old seedlings were incubated in 12-well plates using half-strength liquid MS, pH 5.8 supplemented with $20 \mu \mathrm{M}$ Wortmannin (WM) (Sigma-Aldrich) or $50 \mu \mathrm{M}$ of brefeldin A (BFA) (Sigma-Aldrich). For mock treatment, an equivalent volume of DMSO (Sigma-Aldrich) was used. Incubation with inhibitors took place at $22{ }^{\circ} \mathrm{C}$ in the dark for $165 \mathrm{~min}$ (WM) or $120 \mathrm{~min}$ (BFA) before imaging. FM4-64 staining was performed in half-strength liquid MS, pH 5.8 with $1 \mu \mathrm{M}$ FM4-64 (Molecular probes) for $20 \mathrm{~min}$. Seedlings were imaged with CLSM using $561 \mathrm{~nm}$ laser line for excitation and $670-750 \mathrm{~nm}$ range for emission detection.

\section{Supplemental Material}


Figure S1. Alignment of RLP44 amino acid sequences from various plant species.

Figure S2. Proteomics analysis of RLP44-GFP during transient expression in N. benthamiana and trypsin digestion.

Figure S3. RLP44-GFP WT and Pmimic, but not RLP44-GFP Pdead restore agravitropic growth of cnu2 along the surface of agar plates in the dark as observed in PMElox.

Figure S4. Complementation of cnu2 by RLP44-GFP Wt and p35S:RLP44-GFP Pmimic, but not by p35S:RLP44-GFP Pdead.

Figure S5. RLP44-GFP Pdead is unable to complement the petiole phenotype of rlp44 ${ }^{\text {cnu2 }}$.

Figure S6. Transient expression in N. benthamiana of p35S RLP44-GFP WT, p35S RLP44GFP Pdead, and p35S RLP44-GFP Pmimic leads to plasma membrane localization.

Table S1. Mutants and transgenic lines used in this study.

Table S2. Oligonucleotides used in this study.

Table S3. Overview of constructs generated with GreenGate cloning.

\section{Acknowledgements}

We would like to thank Yi Wu and Pengcheng Wang for advice and technical help with proteomics, Clara Sanchez Rodriguez for sharing seeds of the amiTPL line; Heike Steinininger for technical assistance; Falco Krüger and the Schumacher lab for help with microscopy; and Klaus Harter, Karin Schumacher, Thomas Greb, and Jan Lohmann for discussion. Research in the Wolf lab is supported by the German Research Foundation (DFG) through grants WO 1660/2-1 and SW 1660/6-1. SW is supported by the DFG through the Emmy Noether Programme.

\section{References}

Bakker, J., Spits, M., Neefjes, J. and Berlin, I. (2017) The EGFR odyssey - from activation to destruction in space and time. J Cell Sci, 130, 4087-4096.

Belkhadir, Y. and Jaillais, Y. (2015) The molecular circuitry of brassinosteroid signaling. New Phytol, 206, 522-540.

Beltrao, P., Albanese, V., Kenner, L.R., Swaney, D.L., Burlingame, A., Villen, J., Lim, W.A., Fraser, J.S., Frydman, J. and Krogan, N.J. (2012) Systematic functional prioritization of protein posttranslational modifications. Cell, 150, 413-425.

Brutus, A., Sicilia, F., Macone, A., Cervone, F. and De Lorenzo, G. (2010) A domain swap approach reveals a role of the plant wall-associated kinase 1 (WAK1) as a receptor of oligogalacturonides. Proc Natl Acad Sci U S A, 107, 9452-9457.

Bucherl, C.A., Jarsch, I.K., Schudoma, C., Segonzac, C., Mbengue, M., Robatzek, S., MacLean, D., Ott, T. and Zipfel, C. (2017) Plant immune and growth receptors share common signalling components but localise to distinct plasma membrane nanodomains. elife, 6 .

Cabantous, S., Terwilliger, T.C. and Waldo, G.S. (2005) Protein tagging and detection with engineered self-assembling fragments of green fluorescent protein. Nature biotechnology, 23, 102-107. 
Chaiwanon, J. and Wang, Z.Y. (2015) Spatiotemporal brassinosteroid signaling and antagonism with auxin pattern stem cell dynamics in Arabidopsis roots. Curr Biol, 25, 1031-1042.

Cheon, J., Fujioka, S., Dilkes, B.P. and Choe, S. (2013) Brassinosteroids regulate plant growth through distinct signaling pathways in Selaginella and Arabidopsis. PLoS One, 8, e81938.

Couto, D., Niebergall, R., Liang, X., Bucherl, C.A., Sklenar, J., Macho, A.P., Ntoukakis, V., Derbyshire, P., Altenbach, D., Maclean, D., Robatzek, S., Uhrig, J., Menke, F., Zhou, J.M. and Zipfel, C. (2016) The Arabidopsis Protein Phosphatase PP2C38 Negatively Regulates the Central Immune Kinase BIK1. PLoS Pathog, 12, e1005811.

Couto, D. and Zipfel, C. (2016) Regulation of pattern recognition receptor signalling in plants. Nat Rev Immunol, 16, 537-552.

Dettmer, J., Hong-Hermesdorf, A., Stierhof, Y.D. and Schumacher, K. (2006) Vacuolar H+-ATPase activity is required for endocytic and secretory trafficking in Arabidopsis. Plant Cell, 18, 715730.

Di Rubbo, S., Irani, N.G., Kim, S.Y., Xu, Z.Y., Gadeyne, A., Dejonghe, W., Vanhoutte, I., Persiau, G., Eeckhout, D., Simon, S., Song, K., Kleine-Vehn, J., Friml, J., De Jaeger, G., Van Damme, D., Hwang, I. and Russinova, E. (2013) The clathrin adaptor complex AP-2 mediates endocytosis of brassinosteroid insensitive1 in Arabidopsis. Plant Cell, 25, 2986-2997.

Domozych, D.S., Sorensen, I., Popper, Z.A., Ochs, J., Andreas, A., Fangel, J.U., Pielach, A., Sacks, C., Brechka, H., Ruisi-Besares, P., Willats, W.G. and Rose, J.K. (2014) Pectin metabolism and assembly in the cell wall of the charophyte green alga Penium margaritaceum. Plant Physiol, $165,105-118$.

Dubeaux, G. and Vert, G. (2017) Zooming into plant ubiquitin-mediated endocytosis. Curr Opin Plant Biol, 40, 56-62.

Dubeaux, G., Zelazny, E. and Vert, G. (2015) Getting to the root of plant iron uptake and cell-cell transport: Polarity matters! Commun Integr Biol, 8, e1038441.

Emans, N., Zimmermann, S. and Fischer, R. (2002) Uptake of a fluorescent marker in plant cells is sensitive to brefeldin A and wortmannin. Plant Cell, 14, 71-86.

Erwig, J., Ghareeb, H., Kopischke, M., Hacke, R., Matei, A., Petutschnig, E. and Lipka, V. (2017) Chitininduced and CHITIN ELICITOR RECEPTOR KINASE1 (CERK1) phosphorylation-dependent endocytosis of Arabidopsis thaliana LYSIN MOTIF-CONTAINING RECEPTOR-LIKE KINASE5 (LYK5). New Phytol, 215, 382-396.

Friedrichsen, D.M., Joazeiro, C.A., Li, J., Hunter, T. and Chory, J. (2000) Brassinosteroid-insensitive-1 is a ubiquitously expressed leucine-rich repeat receptor serine/threonine kinase. Plant Physiol, 123, 1247-1256.

Fritz-Laylin, L.K., Krishnamurthy, N., Tor, M., Sjolander, K.V. and Jones, J.D. (2005) Phylogenomic analysis of the receptor-like proteins of rice and Arabidopsis. Plant Physiol, 138, 611-623.

Gadeyne, A., Sanchez-Rodriguez, C., Vanneste, S., Di Rubbo, S., Zauber, H., Vanneste, K., Van Leene, J., De Winne, N., Eeckhout, D., Persiau, G., Van De Slijke, E., Cannoot, B., Vercruysse, L., Mayers, J.R., Adamowski, M., Kania, U., Ehrlich, M., Schweighofer, A., Ketelaar, T., Maere, S., Bednarek, S.Y., Friml, J., Gevaert, K., Witters, E., Russinova, E., Persson, S., De Jaeger, G. and Van Damme, D. (2014) The TPLATE adaptor complex drives clathrin-mediated endocytosis in plants. Cell, 156, 691-704.

Geldner, N., Anders, N., Wolters, H., Keicher, J., Kornberger, W., Muller, P., Delbarre, A., Ueda, T., Nakano, A. and Jurgens, G. (2003) The Arabidopsis GNOM ARF-GEF mediates endosomal recycling, auxin transport, and auxin-dependent plant growth. Cell, 112, 219-230.

Geldner, N. and Jurgens, G. (2006) Endocytosis in signalling and development. Curr Opin Plant Biol, 9, 589-594.

Grebe, M., Xu, J., Mobius, W., Ueda, T., Nakano, A., Geuze, H.J., Rook, M.B. and Scheres, B. (2003) Arabidopsis sterol endocytosis involves actin-mediated trafficking via ARA6-positive early endosomes. Curr Biol, 13, 1378-1387. 
Grefen, C. and Blatt, M.R. (2012) A 2in1 cloning system enables ratiometric bimolecular fluorescence complementation (rBiFC). Biotechniques, 53, 311-314.

Gronnier, J., Crowet, J.M., Habenstein, B., Nasir, M.N., Bayle, V., Hosy, E., Platre, M.P., Gouguet, P., Raffaele, S., Martinez, D., Grelard, A., Loquet, A., Simon-Plas, F., Gerbeau-Pissot, P., Der, C., Bayer, E.M., Jaillais, Y., Deleu, M., Germain, V., Lins, L. and Mongrand, S. (2017) Structural basis for plant plasma membrane protein dynamics and organization into functional nanodomains. elife, 6.

Gust, A.A. and Felix, G. (2014) Receptor like proteins associate with SOBIR1-type of adaptors to form bimolecular receptor kinases. Curr Opin Plant Biol, 21C, 104-111.

Haglund, K. and Dikic, I. (2012) The role of ubiquitylation in receptor endocytosis and endosomal sorting. J Cell Sci, 125, 265-275.

Hartmann, J., Stuhrwohldt, N., Dahlke, R.I. and Sauter, M. (2013) Phytosulfokine control of growth occurs in the epidermis, is likely to be non-cell autonomous and is dependent on brassinosteroids. Plant J, 73, 579-590.

He, Z., Wang, Z.Y., Li, J., Zhu, Q., Lamb, C., Ronald, P. and Chory, J. (2000) Perception of brassinosteroids by the extracellular domain of the receptor kinase BRI1. Science, 288, 23602363.

Hohmann, U., Lau, K. and Hothorn, M. (2017) The Structural Basis of Ligand Perception and Signal Activation by Receptor Kinases. Annu Rev Plant Biol, 68, 109-137.

Hohmann, U., Santiago, J., Nicolet, J., Olsson, V., Spiga, F.M., Hothorn, L.A., Butenko, M.A. and Hothorn, M. (2018) Mechanistic basis for the activation of plant membrane receptor kinases by SERK-family coreceptors. Proc Natl Acad Sci U S A, 115, 3488-3493.

Holt, L.J., Tuch, B.B., Villen, J., Johnson, A.D., Gygi, S.P. and Morgan, D.O. (2009) Global analysis of Cdk1 substrate phosphorylation sites provides insights into evolution. Science, 325, 16821686.

Holzwart, E., Huerta, A.I., Glockner, N., Garnelo Gomez, B., Wanke, F., Augustin, S., Askani, J.C., Schurholz, A.K., Harter, K. and Wolf, S. (2018a) BRI1 controls vascular cell fate in the Arabidopsis root through RLP44 and phytosulfokine signaling. Proc Natl Acad Sci U S A, 115, 11838-11843.

Holzwart, E., Huerta, A.I., Glockner, N., Garnelo Gomez, B., Wanke, F., Augustin, S., Askani, J.C., Schurholz, A.K., Harter, K. and Wolf, S. (2018b) BRI1 controls vascular cell fate in the Arabidopsis root through RLP44 and phytosulfokine signaling. Proc Natl Acad Sci U S A.

Howe, C.L. and Mobley, W.C. (2004) Signaling endosome hypothesis: A cellular mechanism for long distance communication. J Neurobiol, 58, 207-216.

Hunter, T. (2007) The age of crosstalk: phosphorylation, ubiquitination, and beyond. Molecular cell, 28, 730-738.

Jaillais, Y., Belkhadir, Y., Balsemao-Pires, E., Dangl, J.L. and Chory, J. (2011a) Extracellular leucinerich repeats as a platform for receptor/coreceptor complex formation. Proc Natl Acad Sci U S A, 108, 8503-8507.

Jaillais, Y., Hothorn, M., Belkhadir, Y., Dabi, T., Nimchuk, Z.L., Meyerowitz, E.M. and Chory, J. (2011b) Tyrosine phosphorylation controls brassinosteroid receptor activation by triggering membrane release of its kinase inhibitor. Genes Dev, 25, 232-237.

Jamieson, P.A., Shan, L. and He, P. (2018) Plant cell surface molecular cypher: Receptor-like proteins and their roles in immunity and development. Plant science : an international journal of experimental plant biology, 274, 242-251.

Jarsch, I.K., Konrad, S.S., Stratil, T.F., Urbanus, S.L., Szymanski, W., Braun, P., Braun, K.H. and Ott, T. (2014) Plasma Membranes Are Subcompartmentalized into a Plethora of Coexisting and Diverse Microdomains in Arabidopsis and Nicotiana benthamiana. Plant Cell, 26, 1698-1711.

Karimi, M., Inze, D. and Depicker, A. (2002) GATEWAY vectors for Agrobacterium-mediated plant transformation. Trends Plant Sci, 7, 193-195. 
Kutschmar, A., Rzewuski, G., Stuhrwohldt, N., Beemster, G.T., Inze, D. and Sauter, M. (2009) PSKalpha promotes root growth in Arabidopsis. New Phytol, 181, 820-831.

Ladwig, F., Dahlke, R.I., Stuhrwohldt, N., Hartmann, J., Harter, K. and Sauter, M. (2015) Phytosulfokine Regulates Growth in Arabidopsis through a Response Module at the Plasma Membrane That Includes CYCLIC NUCLEOTIDE-GATED CHANNEL17, H+-ATPase, and BAK1. Plant Cell, 27, 1718-1729.

Lampropoulos, A., Sutikovic, Z., Wenzl, C., Maegele, I., Lohmann, J.U. and Forner, J. (2013) GreenGate---a novel, versatile, and efficient cloning system for plant transgenesis. PLoS One, 8, e83043.

Li, J. and Chory, J. (1997) A putative leucine-rich repeat receptor kinase involved in brassinosteroid signal transduction. Cell, 90, 929-938.

Li, J., Wen, J., Lease, K.A., Doke, J.T., Tax, F.E. and Walker, J.C. (2002) BAK1, an Arabidopsis LRR receptor-like protein kinase, interacts with BRI1 and modulates brassinosteroid signaling. Cell, 110, 213-222.

Lin, W., Lu, D., Gao, X., Jiang, S., Ma, X., Wang, Z., Mengiste, T., He, P. and Shan, L. (2013) Inverse modulation of plant immune and brassinosteroid signaling pathways by the receptor-like cytoplasmic kinase BIK1. Proc Natl Acad Sci U S A, 110, 12114-12119.

Lu, D., Lin, W., Gao, X., Wu, S., Cheng, C., Avila, J., Heese, A., Devarenne, T.P., He, P. and Shan, L. (2011) Direct ubiquitination of pattern recognition receptor FLS2 attenuates plant innate immunity. Science, 332, 1439-1442.

Ma, X., Xu, G., He, P. and Shan, L. (2016) SERKing Coreceptors for Receptors. Trends Plant Sci.

Magliery, T.J., Wilson, C.G., Pan, W., Mishler, D., Ghosh, I., Hamilton, A.D. and Regan, L. (2005) Detecting protein-protein interactions with a green fluorescent protein fragment reassembly trap: scope and mechanism. Journal of the American Chemical Society, 127, 146-157.

Martins, S., Dohmann, E.M., Cayrel, A., Johnson, A., Fischer, W., Pojer, F., Satiat-Jeunemaitre, B., Jaillais, Y., Chory, J., Geldner, N. and Vert, G. (2015) Internalization and vacuolar targeting of the brassinosteroid hormone receptor BRI1 are regulated by ubiquitination. Nature communications, 6, 6151.

Matsubayashi, Y., Ogawa, M., Kihara, H., Niwa, M. and Sakagami, Y. (2006) Disruption and overexpression of Arabidopsis phytosulfokine receptor gene affects cellular longevity and potential for growth. Plant Physiol, 142, 45-53.

Matsubayashi, Y., Ogawa, M., Morita, A. and Sakagami, Y. (2002) An LRR receptor kinase involved in perception of a peptide plant hormone, phytosulfokine. Science, 296, 1470-1472.

Mbengue, M., Bourdais, G., Gervasi, F., Beck, M., Zhou, J., Spallek, T., Bartels, S., Boller, T., Ueda, T., Kuhn, H. and Robatzek, S. (2016) Clathrin-dependent endocytosis is required for immunity mediated by pattern recognition receptor kinases. Proc Natl Acad Sci U S A, 113, 11034-11039.

Mithoe, S.C. and Menke, F.L. (2018) Regulation of pattern recognition receptor signalling by phosphorylation and ubiquitination. Curr Opin Plant Biol, 45, 162-170.

Monaghan, J., Matschi, S., Shorinola, O., Rovenich, H., Matei, A., Segonzac, C., Malinovsky, F.G., Rathjen, J.P., MacLean, D., Romeis, T. and Zipfel, C. (2014) The Calcium-Dependent Protein Kinase CPK28 Buffers Plant Immunity and Regulates BIK1 Turnover. Cell host \& microbe, 16, 605-615.

Nam, K.H. and Li, J. (2002) BRI1/BAK1, a receptor kinase pair mediating brassinosteroid signaling. Cell, 110, 203-212.

Nimchuk, Z.L., Tarr, P.T., Ohno, C., Qu, X. and Meyerowitz, E.M. (2011) Plant stem cell signaling involves ligand-dependent trafficking of the CLAVATA1 receptor kinase. Curr Biol, 21, 345-352.

Nishi, H., Hashimoto, K. and Panchenko, A.R. (2011) Phosphorylation in protein-protein binding: effect on stability and function. Structure, 19, 1807-1815.

Nishiyama, T., Sakayama, H., de Vries, J., Buschmann, H., Saint-Marcoux, D., Ullrich, K.K., Haas, F.B., Vanderstraeten, L., Becker, D., Lang, D., Vosolsobe, S., Rombauts, S., Wilhelmsson, P.K.I., Janitza, P., Kern, R., Heyl, A., Rumpler, F., Villalobos, L., Clay, J.M., Skokan, R., Toyoda, A., 
Suzuki, Y., Kagoshima, H., Schijlen, E., Tajeshwar, N., Catarino, B., Hetherington, A.J., Saltykova, A., Bonnot, C., Breuninger, H., Symeonidi, A., Radhakrishnan, G.V., Van Nieuwerburgh, F., Deforce, D., Chang, C., Karol, K.G., Hedrich, R., Ulvskov, P., Glockner, G., Delwiche, C.F., Petrasek, J., Van de Peer, Y., Friml, J., Beilby, M., Dolan, L., Kohara, Y., Sugano, S., Fujiyama, A., Delaux, P.M., Quint, M., Theissen, G., Hagemann, M., Harholt, J., Dunand, C., Zachgo, S., Langdale, J., Maumus, F., Van Der Straeten, D., Gould, S.B. and Rensing, S.A. (2018) The Chara Genome: Secondary Complexity and Implications for Plant Terrestrialization. Cell, 174, 448-464 e424.

Offringa, R. and Huang, F. (2013) Phosphorylation-dependent trafficking of plasma membrane proteins in animal and plant cells. Journal of integrative plant biology, 55, 789-808.

Ortiz-Morea, F.A., Savatin, D.V., Dejonghe, W., Kumar, R., Luo, Y., Adamowski, M., Van den Begin, J., Dressano, K., Pereira de Oliveira, G., Zhao, X., Lu, Q., Madder, A., Friml, J., Scherer de Moura, D. and Russinova, E. (2016) Danger-associated peptide signaling in Arabidopsis requires clathrin. Proc Natl Acad Sci U S A, 113, 11028-11033.

Ott, T. (2017) Membrane nanodomains and microdomains in plant-microbe interactions. Curr Opin Plant Biol, 40, 82-88.

Park, C.J., Peng, Y., Chen, X., Dardick, C., Ruan, D., Bart, R., Canlas, P.E. and Ronald, P.C. (2008) Rice $\mathrm{XB15}$, a protein phosphatase $2 \mathrm{C}$, negatively regulates cell death and XA21-mediated innate immunity. PLoS Biol, 6, e231.

Pearlman, S.M., Serber, Z. and Ferrell, J.E., Jr. (2011) A mechanism for the evolution of phosphorylation sites. Cell, 147, 934-946.

Perraki, A., Defalco, T.A., Derbyshire, P., Avila, J., Sere, D., Sklenar, J., Qi, X., Stransfeld, L., Schwessinger, B., Kadota, Y., Macho, A.P., Jiang, S., Couto, D., Torii, K.U., Menke, F.L.H. and Zipfel, C. (2018) Phosphocode-dependent functional dichotomy of a common co-receptor in plant signalling. Nature, 561, 248-252.

Popper, Z.A., Michel, G., Herve, C., Domozych, D.S., Willats, W.G., Tuohy, M.G., Kloareg, B. and Stengel, D.B. (2011) Evolution and diversity of plant cell walls: from algae to flowering plants. Annu Rev Plant Biol, 62, 567-590.

Proseus, T.E. and Boyer, J.S. (2006) Calcium pectate chemistry controls growth rate of Chara corallina. J Exp Bot, 57, 3989-4002.

Riano-Pachon, D.M., Kleessen, S., Neigenfind, J., Durek, P., Weber, E., Engelsberger, W.R., Walther, D., Selbig, J., Schulze, W.X. and Kersten, B. (2010) Proteome-wide survey of phosphorylation patterns affected by nuclear DNA polymorphisms in Arabidopsis thaliana. BMC genomics, 11, 411.

Richter, S., Kientz, M., Brumm, S., Nielsen, M.E., Park, M., Gavidia, R., Krause, C., Voss, U., Beckmann, H., Mayer, U., Stierhof, Y.D. and Jurgens, G. (2014) Delivery of endocytosed proteins to the cell-division plane requires change of pathway from recycling to secretion. elife, 3, e02131.

Robatzek, S., Chinchilla, D. and Boller, T. (2006) Ligand-induced endocytosis of the pattern recognition receptor FLS2 in Arabidopsis. Genes Dev, 20, 537-542.

Sauter, M. (2015) Phytosulfokine peptide signalling. J Exp Bot, 66, 5161-5169.

Shiu, S.H. and Bleecker, A.B. (2001) Receptor-like kinases from Arabidopsis form a monophyletic gene family related to animal receptor kinases. Proc Natl Acad Sci U S A, 98, 10763-10768.

Sigismund, S., Algisi, V., Nappo, G., Conte, A., Pascolutti, R., Cuomo, A., Bonaldi, T., Argenzio, E., Verhoef, L.G., Maspero, E., Bianchi, F., Capuani, F., Ciliberto, A., Polo, S. and Di Fiore, P.P. (2013) Threshold-controlled ubiquitination of the EGFR directs receptor fate. EMBO J, 32, 2140-2157.

Sigismund, S., Confalonieri, S., Ciliberto, A., Polo, S., Scita, G. and Di Fiore, P.P. (2012) Endocytosis and signaling: cell logistics shape the eukaryotic cell plan. Physiol Rev, 92, 273-366.

Singh, A.P. and Savaldi-Goldstein, S. (2015) Growth control: brassinosteroid activity gets context. J Exp Bot, 66, 1123-1132. 
Smakowska-Luzan, E., Mott, G.A., Parys, K., Stegmann, M., Howton, T.C., Layeghifard, M., Neuhold, J., Lehner, A., Kong, J., Grunwald, K., Weinberger, N., Satbhai, S.B., Mayer, D., Busch, W., Madalinski, M., Stolt-Bergner, P., Provart, N.J., Mukhtar, M.S., Zipfel, C., Desveaux, D., Guttman, D.S. and Belkhadir, Y. (2018) An extracellular network of Arabidopsis leucine-rich repeat receptor kinases. Nature, 553, 342-346.

Sun, Y., Fan, X.Y., Cao, D.M., Tang, W., He, K., Zhu, J.Y., He, J.X., Bai, M.Y., Zhu, S., Oh, E., Patil, S., Kim, T.W., Ji, H., Wong, W.H., Rhee, S.Y. and Wang, Z.Y. (2010) Integration of brassinosteroid signal transduction with the transcription network for plant growth regulation in Arabidopsis. Dev Cell, 19, 765-777.

Swaney, D.L., Beltrao, P., Starita, L., Guo, A., Rush, J., Fields, S., Krogan, N.J. and Villen, J. (2013) Global analysis of phosphorylation and ubiquitylation cross-talk in protein degradation. Nature methods, 10, 676-682.

Tyagi, M., Shoemaker, B.A., Bryant, S.H. and Panchenko, A.R. (2009) Exploring functional roles of multibinding protein interfaces. Protein Sci, 18, 1674-1683.

Van Damme, D., Gadeyne, A., Vanstraelen, M., Inze, D., Van Montagu, M.C., De Jaeger, G., Russinova, E. and Geelen, D. (2011) Adaptin-like protein TPLATE and clathrin recruitment during plant somatic cytokinesis occurs via two distinct pathways. Proc Natl Acad Sci U S A, 108, 615-620.

Venne, A.S., Kollipara, L. and Zahedi, R.P. (2014) The next level of complexity: crosstalk of posttranslational modifications. Proteomics, 14, 513-524.

Viotti, C., Bubeck, J., Stierhof, Y.D., Krebs, M., Langhans, M., van den Berg, W., van Dongen, W., Richter, S., Geldner, N., Takano, J., Jurgens, G., de Vries, S.C., Robinson, D.G. and Schumacher, K. (2010) Endocytic and secretory traffic in Arabidopsis merge in the trans-Golgi network/early endosome, an independent and highly dynamic organelle. Plant Cell, 22, 13441357.

Wang, J., Cai, Y., Miao, Y., Lam, S.K. and Jiang, L. (2009) Wortmannin induces homotypic fusion of plant prevacuolar compartments. J Exp Bot, 60, 3075-3083.

Wang, J., Li, H., Han, Z., Zhang, H., Wang, T., Lin, G., Chang, J., Yang, W. and Chai, J. (2015a) Allosteric receptor activation by the plant peptide hormone phytosulfokine. Nature, 525, 265-268.

Wang, L., Li, H., Lv, X., Chen, T., Li, R., Xue, Y., Jiang, J., Jin, B., Baluska, F., Samaj, J., Wang, X. and Lin, J. (2015b) Spatiotemporal Dynamics of the BRI1 Receptor and its Regulation by Membrane Microdomains in Living Arabidopsis Cells. Mol Plant, 8, 1334-1349.

Wolf, S., Hematy, K. and Hofte, H. (2012a) Growth control and cell wall signaling in plants. Annu Rev Plant Biol, 63, 381-407.

Wolf, S., Mravec, J., Greiner, S., Mouille, G. and Hofte, H. (2012b) Plant cell wall homeostasis is mediated by brassinosteroid feedback signaling. Curr Biol, 22, 1732-1737.

Wolf, S., van der Does, D., Ladwig, F., Sticht, C., Kolbeck, A., Schurholz, A.K., Augustin, S., Keinath, N., Rausch, T., Greiner, S., Schumacher, K., Harter, K., Zipfel, C. and Hofte, H. (2014) A receptor-like protein mediates the response to pectin modification by activating brassinosteroid signaling. Proc Natl Acad Sci U S A, 111, 15261-15266.

Zhou, J., Liu, D., Wang, P., Ma, X., Lin, W., Chen, S., Mishev, K., Lu, D., Kumar, R., Vanhoutte, I., Meng, X., He, P., Russinova, E. and Shan, L. (2018) Regulation of Arabidopsis brassinosteroid receptor BRI1 endocytosis and degradation by plant U-box PUB12/PUB13-mediated ubiquitination. Proc Natl Acad Sci U S A, 115, E1906-E1915. 
A

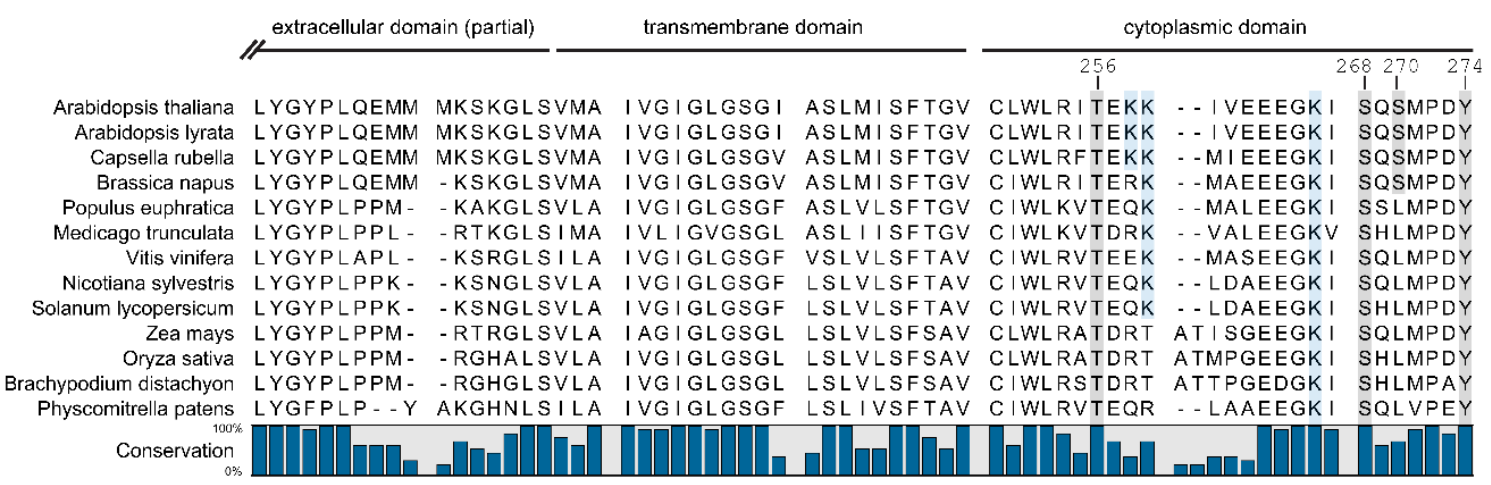

B

C

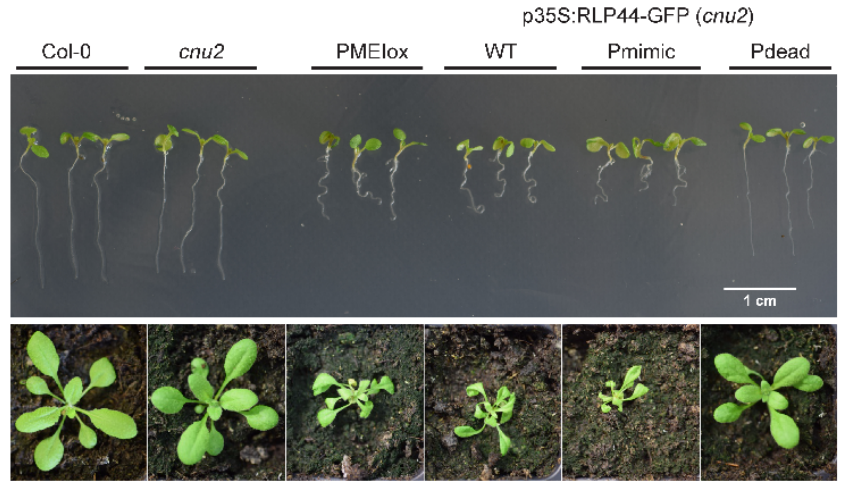

Figure 1. RLP44 has an unusual C-terminal tail with four putative phosphorylation sites that are required for function.

A) The short, cytoplasmic tail of AtRLP44 contains four putative phosphorylation sites, three of which are conserved in RLP44 orthologues. C-terminal part of the extracellular domain, predicted transmembrane domain (http://www.cbs.dtu.dk/services/TMHMM/), and cytoplasmic domain of AtRLP44 are indicated; see Fig. S1 for full alignment.

B) Schematic representation of RLP44 WT, Pdead, and Pmimic variants.

C) Blocking post translational modification of the four putative phosphosites in RLP44-GFP Pdead precludes function in the BR signalling-dependent response to cell wall modification, whereas simulating phosphorylation in RLP44-GFP Pmimic results in RLP44-GFP WT-like functionality. Expression of RLP44-GFP WT and Pmimic, but not of Pdead, is able to complement the PMElox suppressor mutant cnu2 and leads to recovery of the PMElox root waving phenotype in seedlings and contorted leaf arrangement in adult plants. 

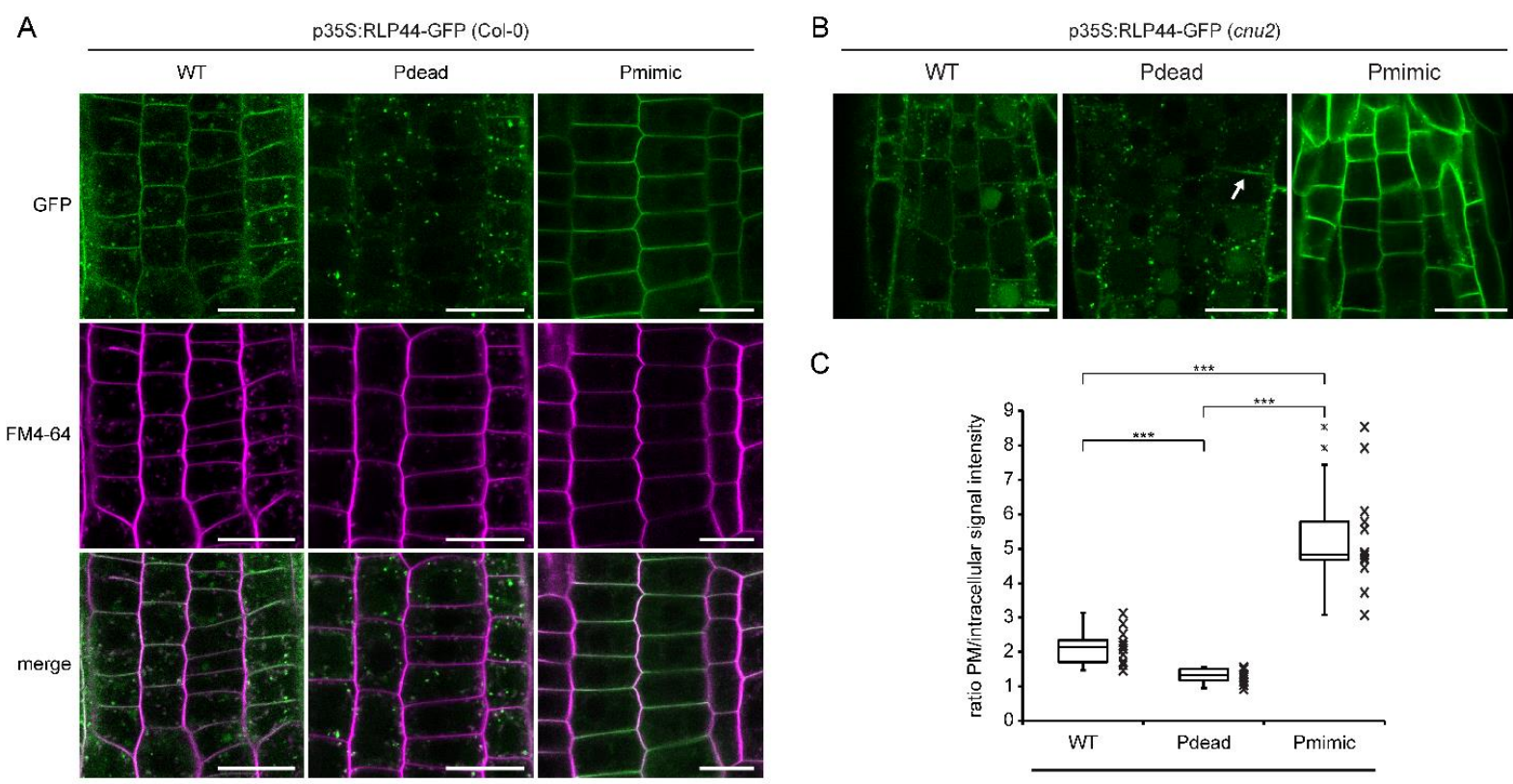

C

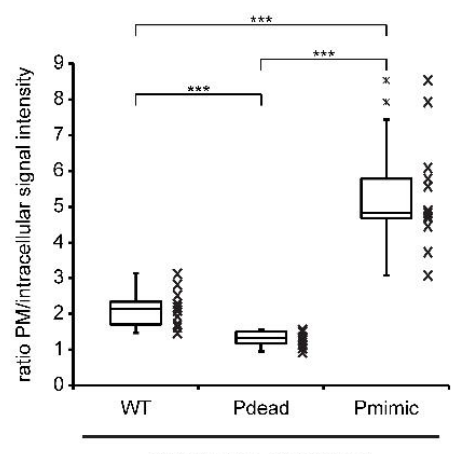

p35S:RLP44-GFP (cnu2)

Figure 2. RLP44-GFP phospho-status determines its subcellular localization.

A) RLP44-GFP WT-derived fluorescence is observed at the plasma membrane and in the endomembrane system, as previously demonstrated (Wolf et al., 2014). RLP44-GFP Pdead is mostly confined to endomembranes, whereas RLP44-GFP Pmimic shows prominent plasma membrane localization. Bars $=20 \mu \mathrm{m}$.

B) The subcellular distribution of RLP44-GFP variants is maintained in the cnu2 background. Arrow points to a fusing cell plate that shows increased RLP44-GFP Pdead fluorescence. Bars $=50 \mu \mathrm{m}$.

C) Quantification of mean plasma membrane to intracellular fluorescence ratio. Boxes indicate range from $25^{\text {th }}$ to $75^{\text {th }}$ percentile, horizontal line indicates the median, whiskers indicate data points within 1.5 times the interquartile range. Markers above whiskers indicate outliers, markers alongside box and whisker diagrams indicate individual data points, $\mathrm{n}=12$ measurements (cells) in 3 independent roots for each genotype. Asterisks indicate statistically significant differences with ${ }^{* * *} \mathrm{p}<0.001$ according to a Student's t-test. 
A

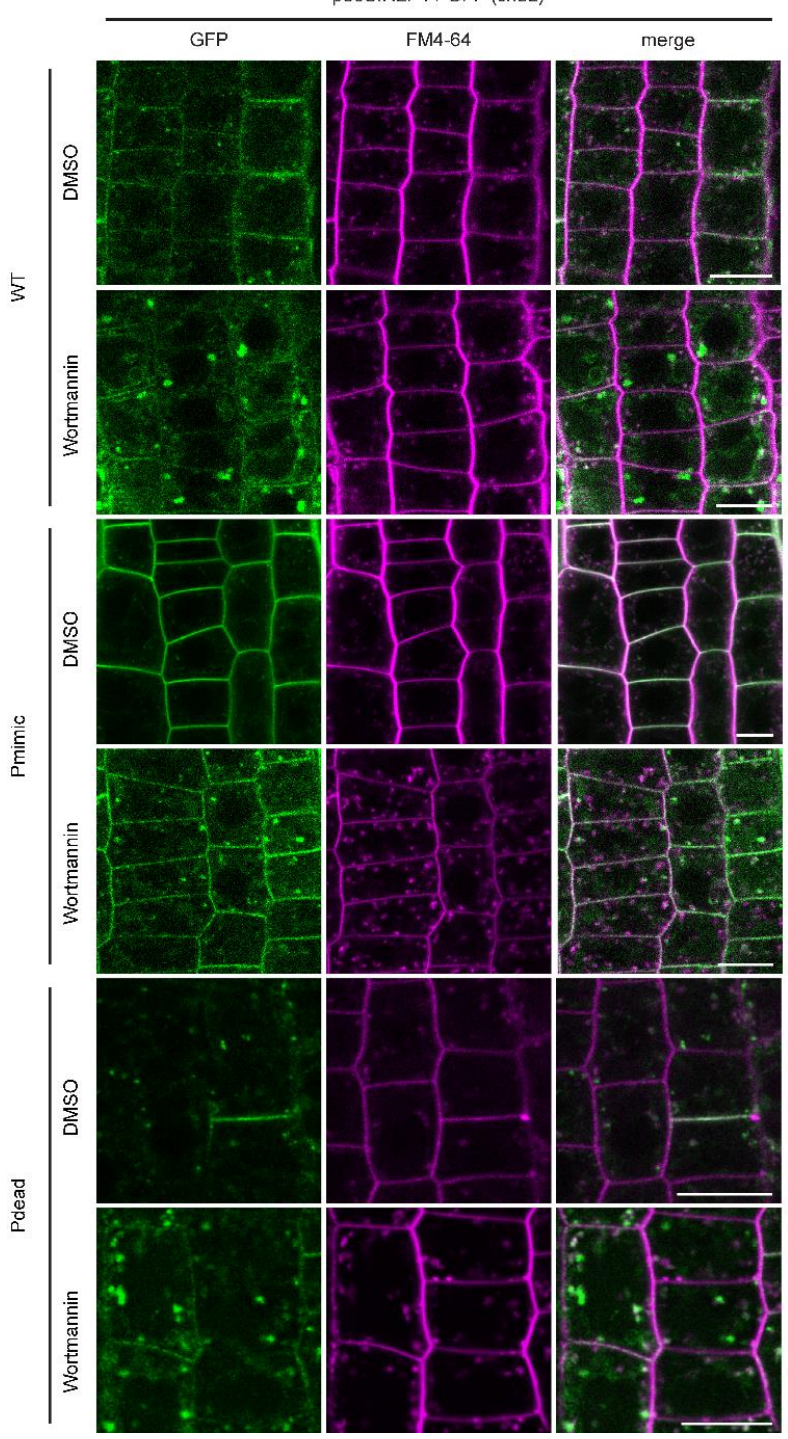

B
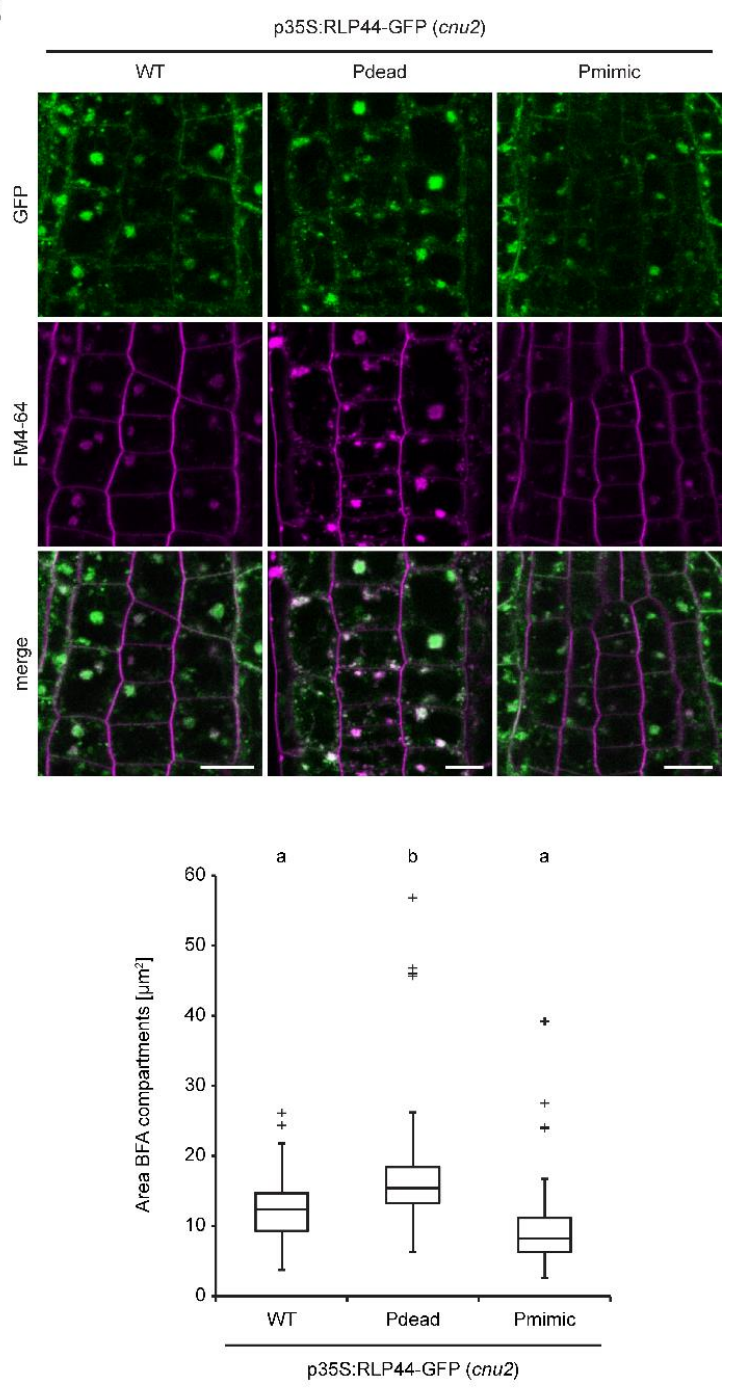

Figure 3. RLP44-GFP variants undergo endocytosis.

A) Fluorescence derived from RLP44-GFP WT, Pdead, and Pmimic variants accumulates in enlarged structures after $20 \mu \mathrm{M}$ Wortmannin treatment for 165 minutes, suggesting they reach late endosomes. Note increased plasma membrane labelling of RLP44-GFP Pdead after WM treatment. Bars $=10 \mu \mathrm{m}$.

B) Fluorescence derived from RLP44-GFP WT, Pdead, and Pmimic variants accumulates in BFA bodies (upper panel). Roots were treated with $50 \mu \mathrm{M}$ of BFA or DMSO for 120 minutes and with FM4-64 for 20 minutes before imaging. Image quantification reveals largest fluorescent area in BFA bodies of RLP44-GFP-derived fluorescence (lower panel), $n=90-116$ measurements (cells) in 18 independent roots for each genotype. Lettering indicates statistically significant difference according to Tukey's post-hoc test following one-way ANOVA. Bars $=10 \mu \mathrm{m}$. 
A

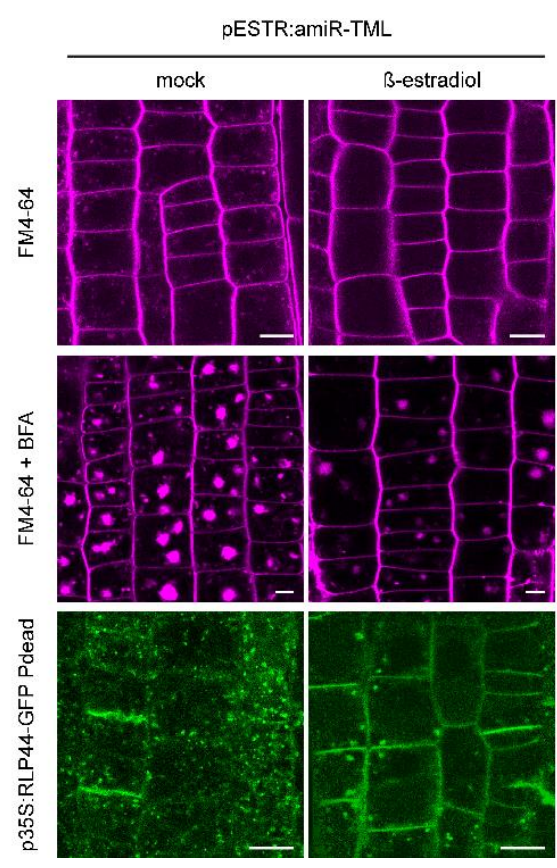

B

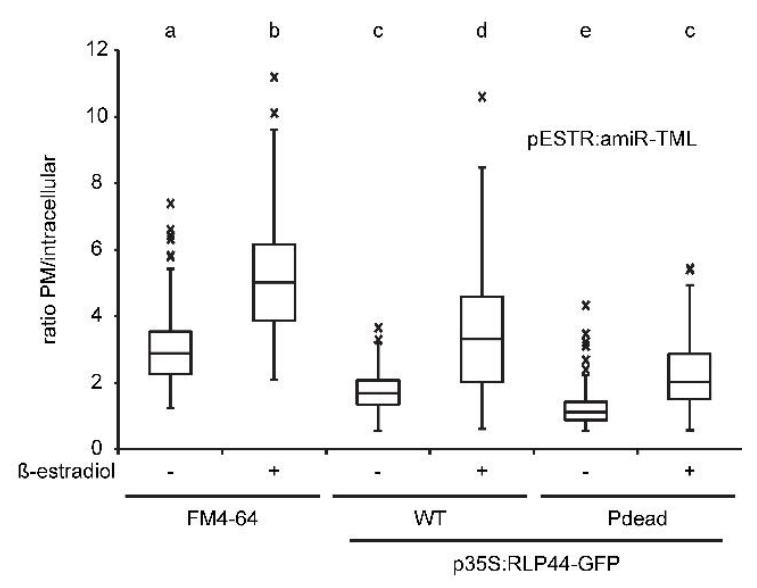

Figure 4. Blocking phosphorylation promotes endocytosis of RLP44-GFP.

A) Inducible expression of amiRNAs targeting the clathrin adapter complex TPLATE leads to reduced formation of endosomes and decreased uptake of FM4-64 into BFA bodies compared to mock control, suggesting efficient blockage of clathrin-mediated endocytosis. Knock-down of TPLATE in RLP44-GFP Pdead leads to enhanced plasma membrane localization. Bars = $10 \mu \mathrm{m}$.

B) Quantification of mean plasma membrane to intracellular fluorescence ratio in the indicated genotypes with and without amiRNA-mediated knock down of TPLATE. Boxes indicate range from $25^{\text {th }}$ to $75^{\text {th }}$ percentile, horizontal line indicates the median, whiskers indicate data points within 1.5 time the interquartile range. Markers above whiskers indicate outliers, $n=78-104$ measurements from 15-18 independent roots each. Letters indicate statistically significant differences according to Tukey's post hoc test after one-way ANOVA. 


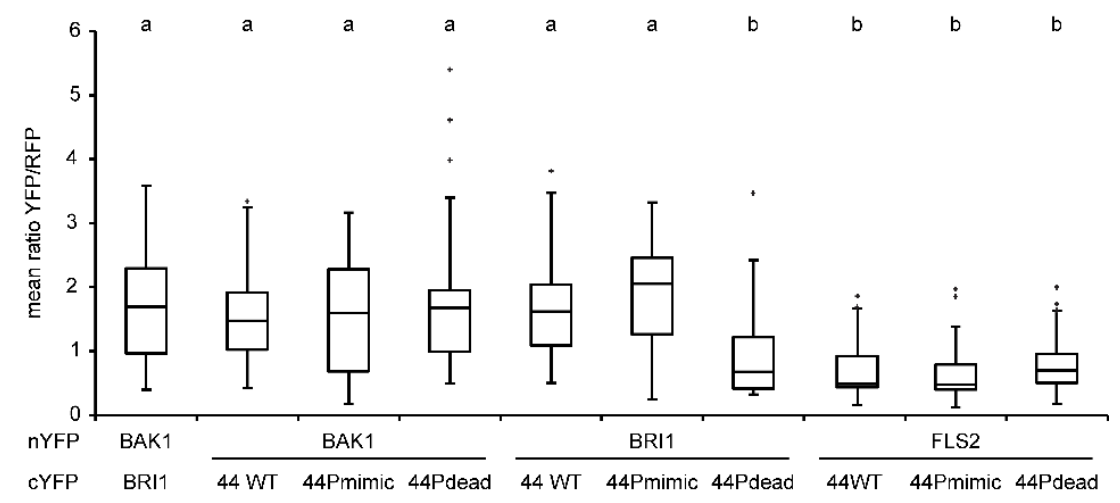

Figure 5. RLP44 phosphosites are required for interaction with BRI1.

Ratiometric bimolecular fluorescence complementation in $N$. benthamiana leaves demonstrates that all three RLP44-GFP variants are able to interact with BAK1, but only WT and Pmimic interact with BRI1. The BAK1-BRI1 interaction is used as a positive control, FLS2 is used as negative control. Boxes indicate mean fluorescent ratio ranging from first to third quartile, horizontal line indicates median. Whiskers indicate data within 1.5 times the interquartile range, crosses denote outliers. Lower case letters indicate statistically significant difference with $\mathrm{P}<0.05$ determined by Tukey's post hoc test after one-way ANOVA, $n=26-94$ independent measurements (images) taken from at least three transformed leaves. 
A

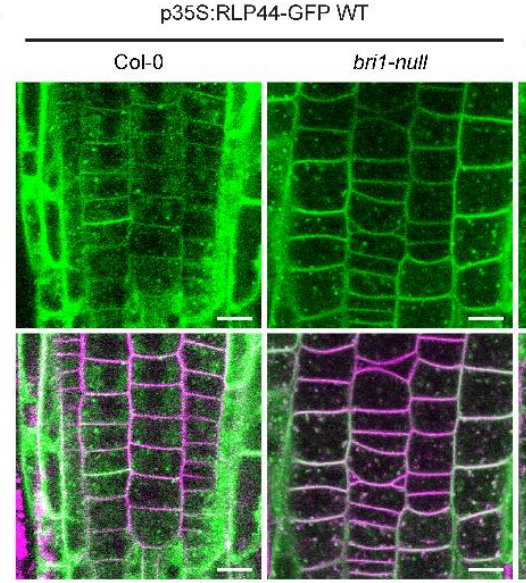

p35S:RLP44-GFP Pdead

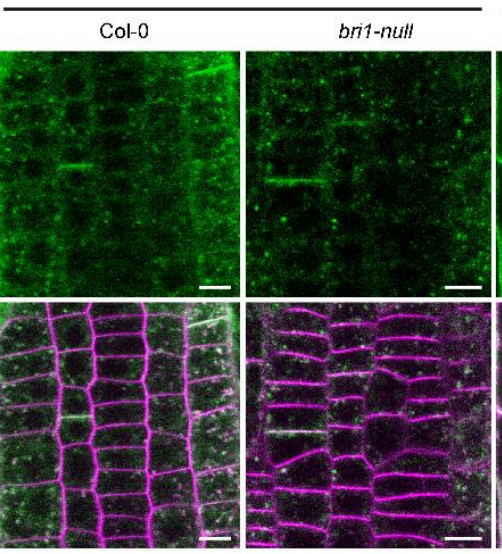

p35S:RLP44-GFP Pmimic

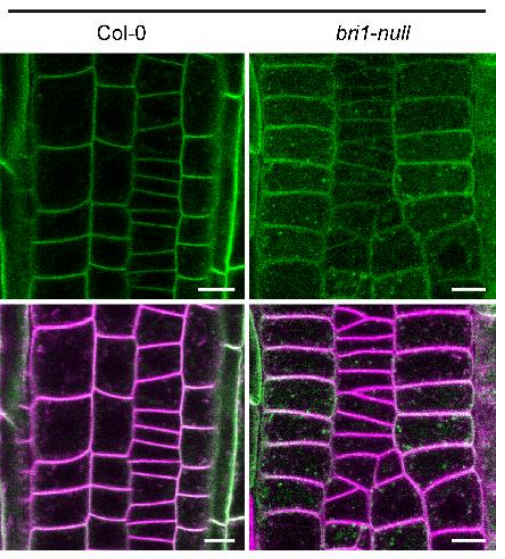

B

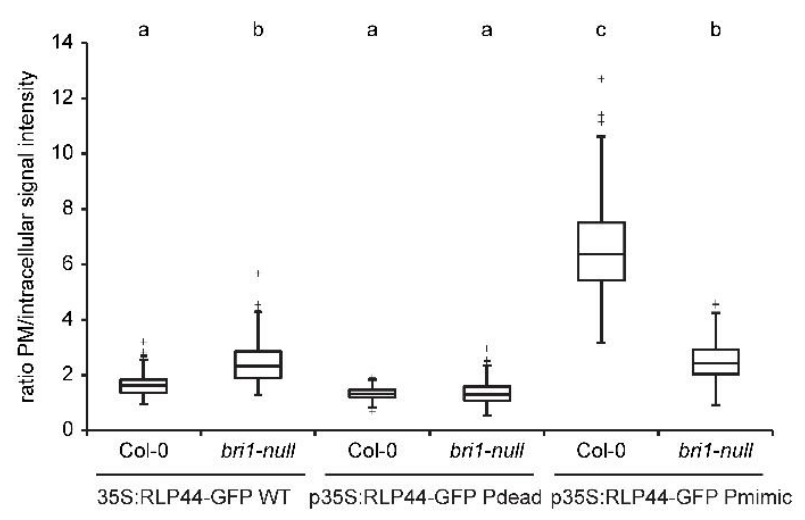

Figure 6. Subcellular distribution of RLP44-GFP depends on BRI1.

A) Comparison of fluorescence distribution derived from the three RLP44-GFP variants in Col0 and bri1-null background. Note the increased appearance of intracellular fluorescence in RLP44-GFP Pmimic expressed in the bri1-null mutant. Bars $=5 \mu \mathrm{m}$.

B) Quantification of mean plasma membrane to intracellular fluorescence ratio in the indicated genotypes, derived from images as in A). Boxes indicate range from $25^{\text {th }}$ to $75^{\text {th }}$ percentile, horizontal line indicates the median, whiskers indicate data points within 1.5 time the interquartile range. Markers above whiskers indicate outliers, $n=48-62$ cells from 12 independent roots for each genotype. Letters indicate statistically significant differences according to Tukey's post hoc test after one-way ANOVA. 
A

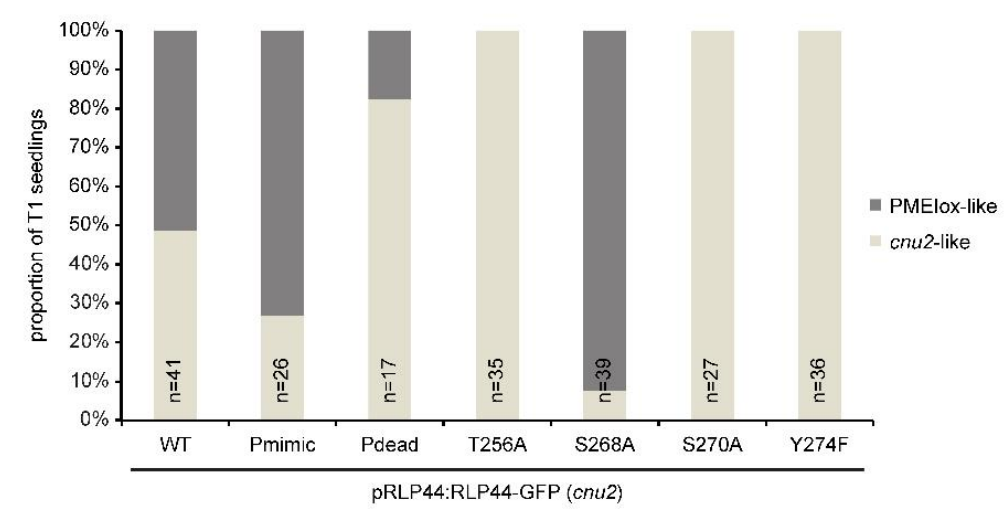

B

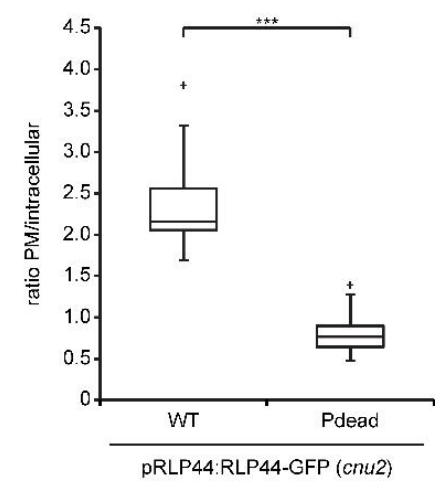

C PRLP44:RLP44-GFP (cnu2)

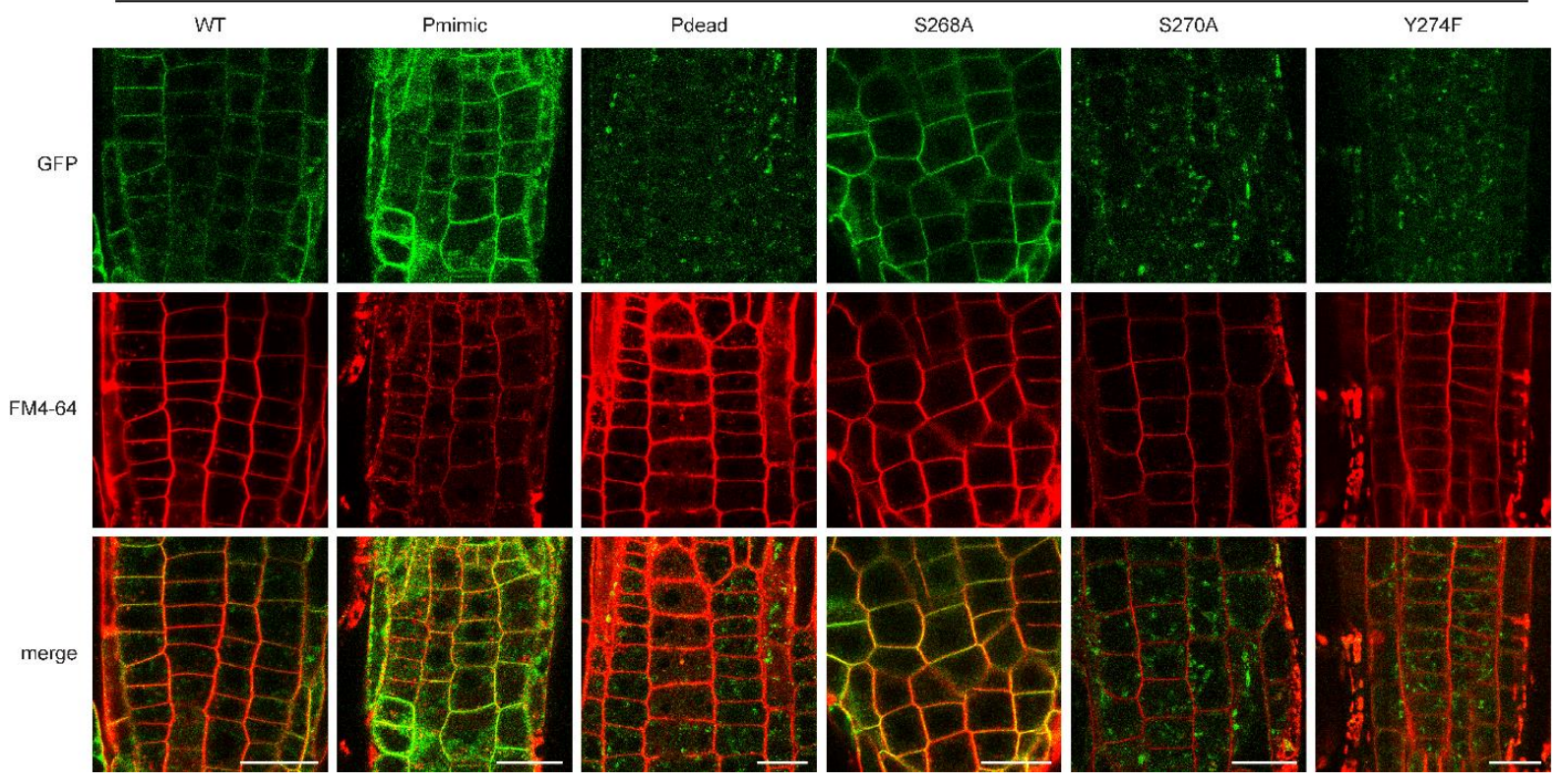

Figure 7. Individual phospho-sites have opposing effects on RLP44 function in BR signalling activation.

A) Proportion of cnu2 seedlings transformed with the indicated pRLP44:RLP44-GFP mutant constructs that are complemented (PMElox-like) and non-complemented (cnu2-like). Total number of transgenic seedlings is indicated.

$B)$ The decrease in the ratio of plasma membrane to intracellular fluorescence is maintained when RLP44-GFP Pdead is expressed under control of the RLP44 promoter.

C) Subcellular fluorescence distribution derived from the indicated $p R L P 44: R L P 44-G F P$ (cnu2) variants, demonstrating that the increased activity of the S268A mutant $(A)$ is correlated with enhanced plasma membrane localization. Bars $=20 \mu \mathrm{m}$. 
A

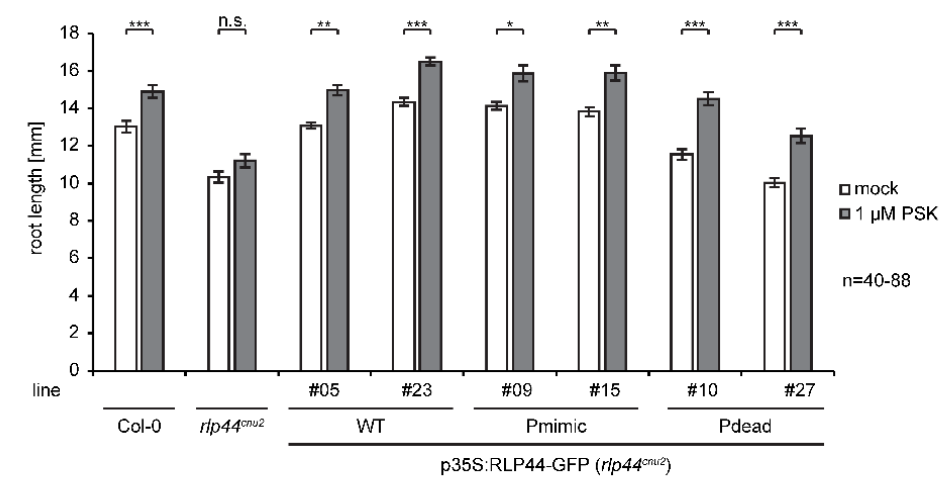

$\mathrm{C}$

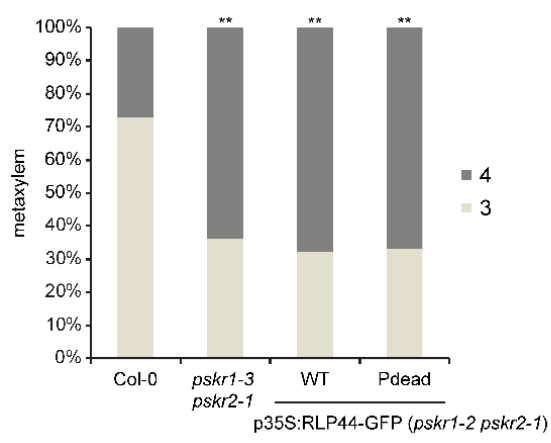

B
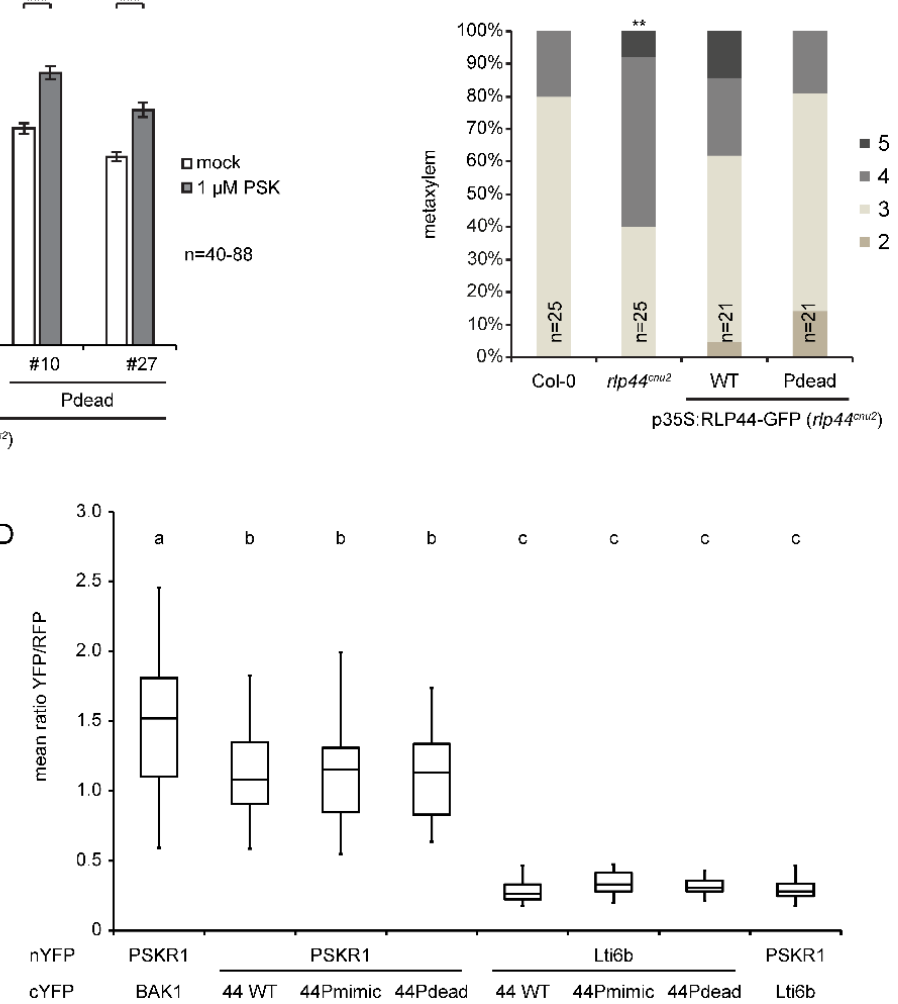

Figure 8. RLP44 is functional in PSK signalling irrespective of potential phospho-status.

A) Root length response to exogenous application of PSK peptide is impaired in rlp44 $4^{\text {cnu2 }}$ (Holzwart et al., 2018), but restored by expression of all three RLP44-GFP variants, including Pdead. Bars denote root length after five days of growth on control plates or plates containing $1 \mu \mathrm{M}$ PSK peptide, $\mathrm{n}=40-88$. Asterisks indicate statistical significance according to Tukey's post hoc test after one-way ANOVA with ${ }^{*} \mathrm{P}<0.05$, ${ }^{* *} \mathrm{P}<0.01,{ }^{* * *} \mathrm{P}<0.001$. B) The PSK signalling-dependent ectopic xylem phenotype of $r$ lp44 ${ }^{\text {cnu2 }}$ (Holzwart et al., 2018) is restored by expression of RLP44-GFP Pdead. C) Effect of RLP44 expression depends on the presence of PSK receptors PSKR1 and PSKR2. Bars in B) and C) denote frequency of roots with indicated number of metaxylem cells, asterisks indicate statistically significant difference from Col-0 based on Dunn's post-hoc test with Benjamini-Hochberg correction after Kruskal-Wallis modified U-test ( $\left.{ }^{* *} p<0.01\right)$. D) Ratiometric bimolecular fluorescence complementation in $N$. benthamiana leaves demonstrates that all three RLP44-GFP variants are able to interact with PSKR1. PSKR1-nYFP and BAK1-cYFP serves as positive control, Lti6b serves as negative control. Boxes indicate mean fluorescence ratio ranging from first to third quartile, horizontal line indicates median. Whiskers indicate data within 1.5 times the interquartile range. Lower case letters indicate statistically significant difference with $\mathrm{P}<0.05$ determined by Tukey's post hoc test after one-way ANOVA, $n=30-43$ independent measurements (images) taken from at least three transformed leaves. 